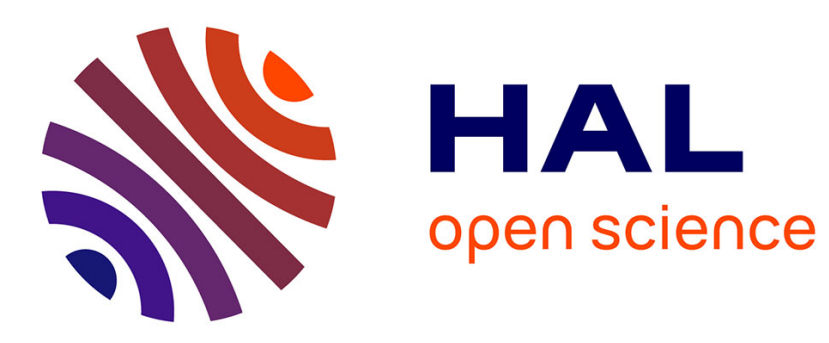

\title{
A multiscale microstructure model of carbon black distribution in rubber
}

Aurélie Jean, Dominique Jeulin, Samuel Forest, Sabine Cantournet, Franck N'Guyen

\section{- To cite this version:}

Aurélie Jean, Dominique Jeulin, Samuel Forest, Sabine Cantournet, Franck N'Guyen. A multiscale microstructure model of carbon black distribution in rubber. Journal of Microscopy, 2011, 241 (3), pp.243-260. 10.1111/j.1365-2818.2010.03428.x . hal-00585338

\section{HAL Id: hal-00585338}

https://hal-mines-paristech.archives-ouvertes.fr/hal-00585338

Submitted on 12 Jul 2018

HAL is a multi-disciplinary open access archive for the deposit and dissemination of scientific research documents, whether they are published or not. The documents may come from teaching and research institutions in France or abroad, or from public or private research centers.
L'archive ouverte pluridisciplinaire HAL, est destinée au dépôt et à la diffusion de documents scientifiques de niveau recherche, publiés ou non, émanant des établissements d'enseignement et de recherche français ou étrangers, des laboratoires publics ou privés. 


\title{
A multiscale microstructure model of carbon black distribution in rubber
}

\author{
A. JEAN*, $\dagger$, D. JEULIN $\dagger$, S. FOREST $*$, S. CANTOURNET* \\ \& F. N'GUYEN* \\ * MINES ParisTech, Centre des Matériaux, CNRS UMR 7633, Evry, France \\ $\dagger$ MINES ParisTech, Centre de Morphologie Mathématique, Mathématiques et Systèmes, \\ Fontainebleau, France
}

Key words. Carbon black, Cox Boolean model, image analysis, mathematical morphology, multiscale distribution, nanocomposites, percolation.

\section{Summary}

The increase of observations and computational capabilities favoured the numerical simulation of microstructure to derive the effective properties of materials. Indeed, the multiscale approaches, that use homogenization techniques, enable us to estimate or to give bounds of the overall properties of heterogeneous media. In this work, the objective is to develop a three-dimensional mathematical model of the morphology of the microstructure of rubber composite containing carbon black nano-fillers. This multiscale model consists of a combination of some primary models that correspond to the physical scales of the microstructure. It is identified according to an original method that uses statistical moments from experimental transmission electronic microscope (TEM) image data and from numerical TEM simulations. This method leads to three-dimensional representative simulations of microstructures that take the complex clustering effect of particles in aggregates, into account. Finally, the identified model of the morphology satisfies the experimental percolation rate of the carbon black aggregates in the material.

\section{Introduction}

The extensive possibilities of three-dimensional observations of microstructure provide much information about their morphology, including the nature and the fraction of the phases in the microstructure and their spatial distribution. The tremendous increase of computational capabilities enables us to compute large three-dimensional simulated microstructures to derive the effective physical properties

Correspondence to: Aurelie Jean, Ph.D., Formerly: Centre des Materiaux, Mines ParisTech, France. Current Address: Department of Bioengineering, Penn State University, 223 Hallowell Building, University Park, PA 16802, U.S.A. Tel.: 814865 6746; fax: 814863 0490; e-mail: axj20@psu.edu from the knowledge of the microstructure morphology. This is the multiscale mechanical approach that resorts to homogenization techniques. Two procedures are considered to obtain the three-dimensional microstructures. In the first procedure, three-dimensional information on the morphology is accurate enough to create a finite element model (Madi et al., 2007) from the reconstruction of the isosurface from Marching Cubes technique (Lorensen \& Cline, 1987). In some cases, the high cost of three-dimensional observations or the lack of information on the three-dimensional morphology lead to generate simulated microstructures. In this paper, the microstructure of a rubber with carbon black fillers is modelled using the statistical information about the morphology from TEM observations.

To model a microstructure, two ways can be investigated. The first way consists in modelling the microstructure by a simple morphological model that uses an elementary cell for periodical computations. This method is widely used in two dimensions (Zeman \& Sejnoha, 2001) and can be extended in three dimensions. Indeed, an inclusion located at the centre of a tetrakaidecaedron cell is used to determine the effective behaviour of centred cubic symmetry of fillers in a rubber matrix (Jean, 2009). The second way consists in simulating more realistic three-dimensional microstructures. This approach is motivated by the large information data on the three-dimensional morphology and by the increase of capabilities of computations.

The first three-dimensional models come from the tesselation of space into cells. Indeed, the Voronoi tesselation is widely known and used in the case of polycrystals (Barbe et al., 2001; Osipov et al., 2008; Gérard et al., 2009) and granular media (Sab \& Boumediene, 2005). The tesselation of space into a combination of a tetrakaidecaedron with a dodecaedron cell can model a foam (Weaire, 2008). The second class of models considers inclusions and/or pores in a matrix. For instance in Hain \& Wriggers (2008), a hard core 
spheres model is performed to describe the aggregates in a concrete.

In the case of rubber with fillers, in Bergstrom \& Boyce (1998) the carbon black particles are not described but the aggregates are modelled as squares or dodecaedra in an elastomeric volume, to derive the large deformation behaviour from finite element computations. In Jha et al. (2007), a cuberbille model is considered to describe the aggregates in the rubber matrix with a third phase that represents the bound rubber around the aggregates. In Naito et al. (2007), the aggregates are defined as the union of carbon black spherical-shaped particles. In Laiarinandrasana et al. (2009), an ideal microstructure is also obtained by placing a spherical carbon black particle at the centre of a tetrakaidecaedron cell to compute the visco-elastic behaviour of a centre cubic symmetry composite from periodical finite element computations. In general, all these works favour the use of a three-dimensional microstructural model to take the interaction between phases into account.

The objective of the work is to establish a mathematical model of the morphology of the microstructure to compute virtual microstructures. Many works deal with the multi-scale modelling (Jeulin, 1991) and in the particular case of carbon black composites (Jeulin \& Le Coënt, 1995; Savary et al., 1999; Delarue, 2001; Moreaud \& Jeulin, 2005; Jean et al., 2007). The model is identified according to an original method that uses statistical moments from TEM image data (Jean et al., 2007). To underline the efficiency of the method, two materials with the same volume fraction are studied in this paper. These materials differ by their time of mixing and therefore by their carbon black distribution.

In what follows, the materials used in this study are first introduced (Section 'Introduction'). Then, the segmentation of TEM images and morphological measurements are detailed in Section 'Segmentation of the TEM images'. In the two next sections 'Multiscale model of microstructure' and 'Parameter identification method', the carbon black distribution is described by a multiscale model with a specific identification procedure. Finally, the identified model of the distribution of carbon black is validated using the percolation criteria.

\section{Materials and methods}

\section{Material}

Rubber is used in a wide range of technical products. To improve the physical properties of a rubber, the stiffness for instance, it must be reinforced by nanoscopic fillers. Mostly, these nanoscopic fillers come from silica or carbon black material. This study focuses on rubber with carbon black fillers. The nature of fillers, their volume fraction and the mixing time and velocity have a strong influence on the microstructure of the material. Indeed, the distribution of fillers tends to be more homogeneous with the time of mixing. Furthermore, using a high-volume fraction of fillers tends to create percolating paths of carbon black in the matrix. These continuous networks appear in the microstructure for increasing volume fractions when the time of mixing increases. The phenomena of percolating network and distribution of fillers, and in general the morphology of the microstructure, lead to a specific macroscopic physical behaviour. Indeed, the stiffness and the nonlinearity (Payne, 1962) increase with the volume fraction of fillers whereas they decrease with time.

\section{Microstructure}

Two elastomeric materials, filled with $14 \%$ in volume of carbon black, are considered, called $M_{1}$ and $M_{2}$ corresponding to a low and a high mixing time, respectively. The materials and the TEM images acquisition were made by Michelin (Ladoux Technology Center, France). Figures 1 and 2 show micrographs of these materials obtained by TEM. These images contain 512 pixels on the length with a resolution of $3.2 \mathrm{~nm}$ per pixel. The slices, cut by a microtome under cryogenic conditions, dedicated to the TEM observations have a thickness that is equal to $40 \mathrm{~nm}( \pm 10 \mathrm{~nm})$. There are up to two particles in the thickness and therefore in the final TEM micrographs. A set of about 40 images per material is needed to reach a representative sample, as it will be shown in Section 'Statistical moments and morphological data'.

Qualitatively, for both materials, three scales are observed in the microstructure on TEM images. These scales are the scale of carbon black particles, the scale of aggregates and the scale of the matrix. The spherical particles have a mean radius of

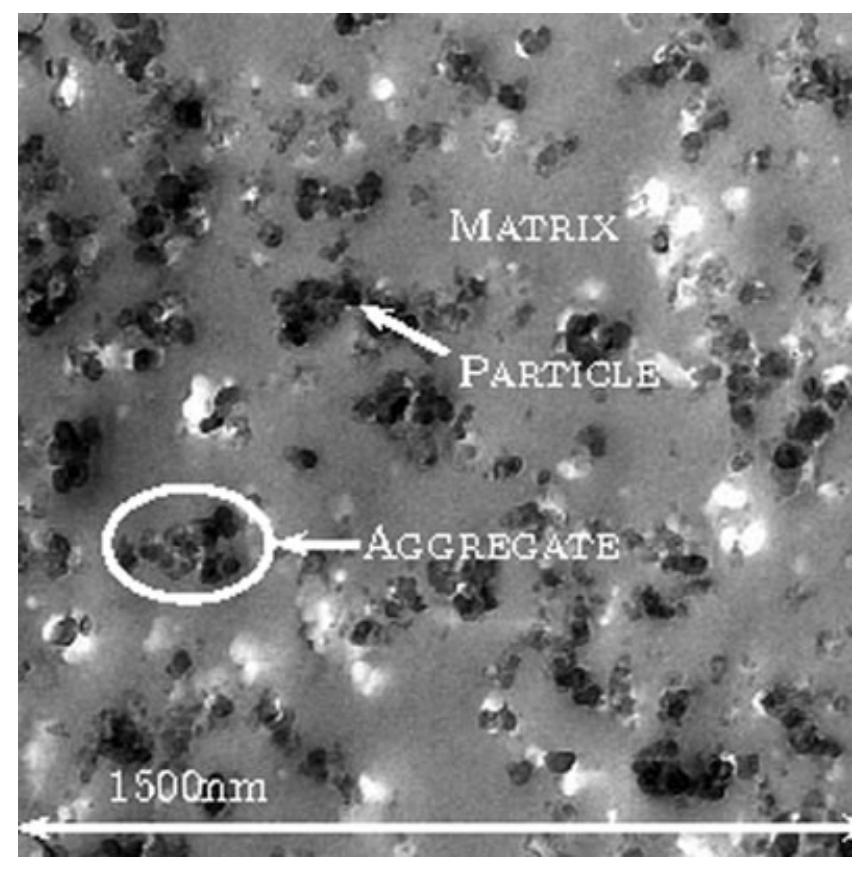

Fig. 1. Observation of filled rubber's microstructure, $M_{1}$ (source: Michelin). 


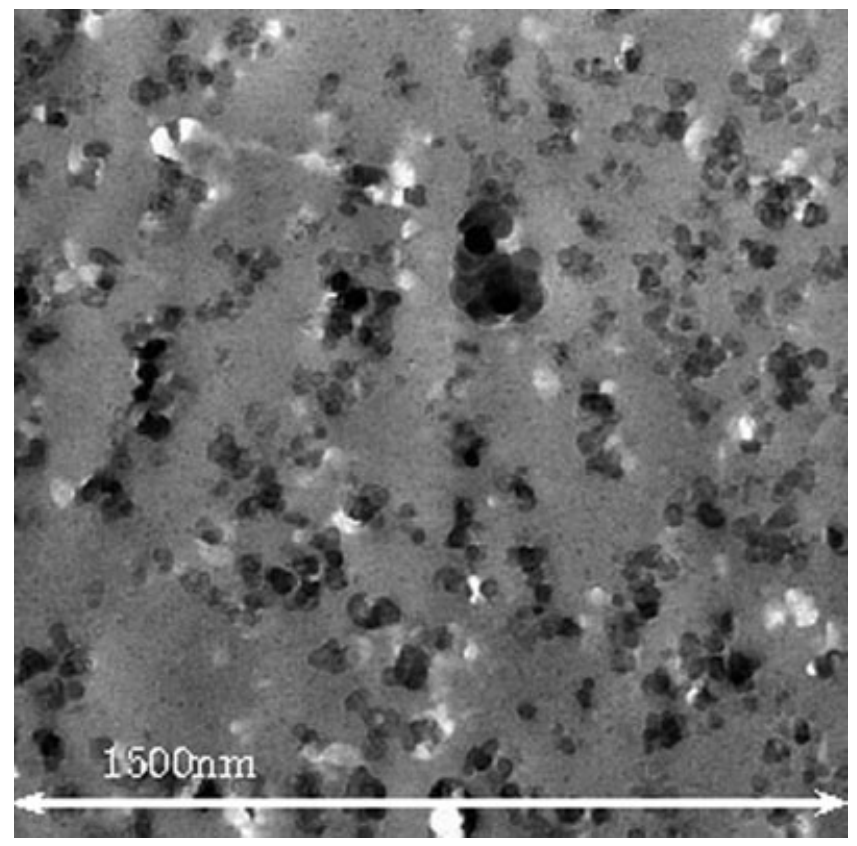

Fig. 2. Observation of filled rubber's microstructure, $M_{2}$ (source: Michelin).

$20 \mathrm{~nm}$. The union of these particles creates aggregates which mean size is around $200 \mathrm{~nm}$. The rubber matrix is located between the aggregates. Compared to material $M_{1}$, the second microstructure seems to be more homogeneous. Indeed, for material $M_{2}$, the mean distance between aggregates is smaller than in the case of material $M_{1}$. To model with precision the morphology of the microstructure, an accurate quantitative description is needed. The next section deals with characterizing the distribution of fillers using a statistical morphological approach.

\section{Segmentation of the TEM images}

This section aims at describing the spatial distribution of particles and aggregates measuring the mean statistical quantities over binary TEM images. The first section is devoted to the presentation of the segmentation algorithm for binarizing TEM images. In the second section, the statistical quantities, retained for the characterization, are introduced. Finally, the obtained mean results are reported, in the case of both materials considered in this paper.

Segmentation algorithm. The segmentation algorithm includes all the operations that are required to transform a grey level image ( 8 bits) into binary one (1 bit). The threshold which is the main operation of this transformation, is chosen to extract a specific part of the initial grey level image. This part corresponds to the white label.

In the case of the TEM images, the set which has to be extracted is the carbon black phase. The white dapples in the grey TEM images are the prints of aggregates which have been taken off by the blade of the microtome. These prints are also introduced in the set to be extracted. The retained algorithm must satisfy several criteria. All the aggregates have to be included in the final binary images. Furthermore, the initial external shape of carbon black aggregates has to be described with precision in the black and white images. The algorithm has to be also robust, to lead to a semi-automatic procedure because of the large number of images to be processed. Indeed, the gradient of the illumination and the difference of contrast at some parts of each individual image, make the segmentation procedure difficult. Similarly, a difference of contrast and illumination between images can be noticed.

Figure 3 illustrates the algorithm developed for the segmentation. A micrograph of material $M_{1}$ is chosen to illustrate each step of the procedure. All these operations are performed using the software Micromorph $\odot(C M M /$ ARMINES/MinesParisTech/TRANSVALOR, 2002). First, a median filter is applied to filter noise in the image (Fig. 4b). Then, two main processes are considered.

The first step aims to extracting the visible black aggregates. A black top hat, by closing with a disc of a radius of 12 pixels, is applied to detect the dark zones in the image (Fig. 4c). Then, two thresholds are applied, independently on the image, with a level around 30 and 50 that detects the darkest and lightest zones, respectively (Figs $4 \mathrm{~d}$ and e). The union of these two images enables us to define the markers that are required for the watershed segmentation (Fig. 4e) to extract the boundary of the visible aggregates (Fig. 4f). A dilatation by an hexagon of one pixel leads to the final binary image that characterizes the visible aggregates (Fig. 5b).

The second step consists in extracting the white prints of aggregates by thresholding the filtered image (Fig. 4a) with a level higher than 185 (Fig. 5c). The union of this image with the last resulting image of visible aggregates leads to the final set of carbon black fillers in the TEM image (Fig. 5d). The values of the threshold level need sometimes to be slightly changed, to take the difference of light and contrast between samples of images into account.

Results. Figures 6 and 7 show examples of TEM micrographs and their corresponding binary images for materials $M_{1}$ and $M_{2}$, respectively. The apparent area fraction of white phase in the present TEM binary image is equal to $28.2 \%$ and $27.6 \%$ for materials $M_{1}$ and $M_{2}$, respectively. This fraction is higher than the initial volume fraction of the material ( $14 \%)$ because the TEM technique leads to observations of thick sections, enlarging the carbon black particles by projection over the thickness.

The shape of aggregates is well described in the binary micrographs but it is still difficult to describe with precision the shape of each individual particle in the aggregate. Nevertheless, this segmentation algorithm enables us to characterize accurately the distribution of carbon black fillers in the polymer matrix. 


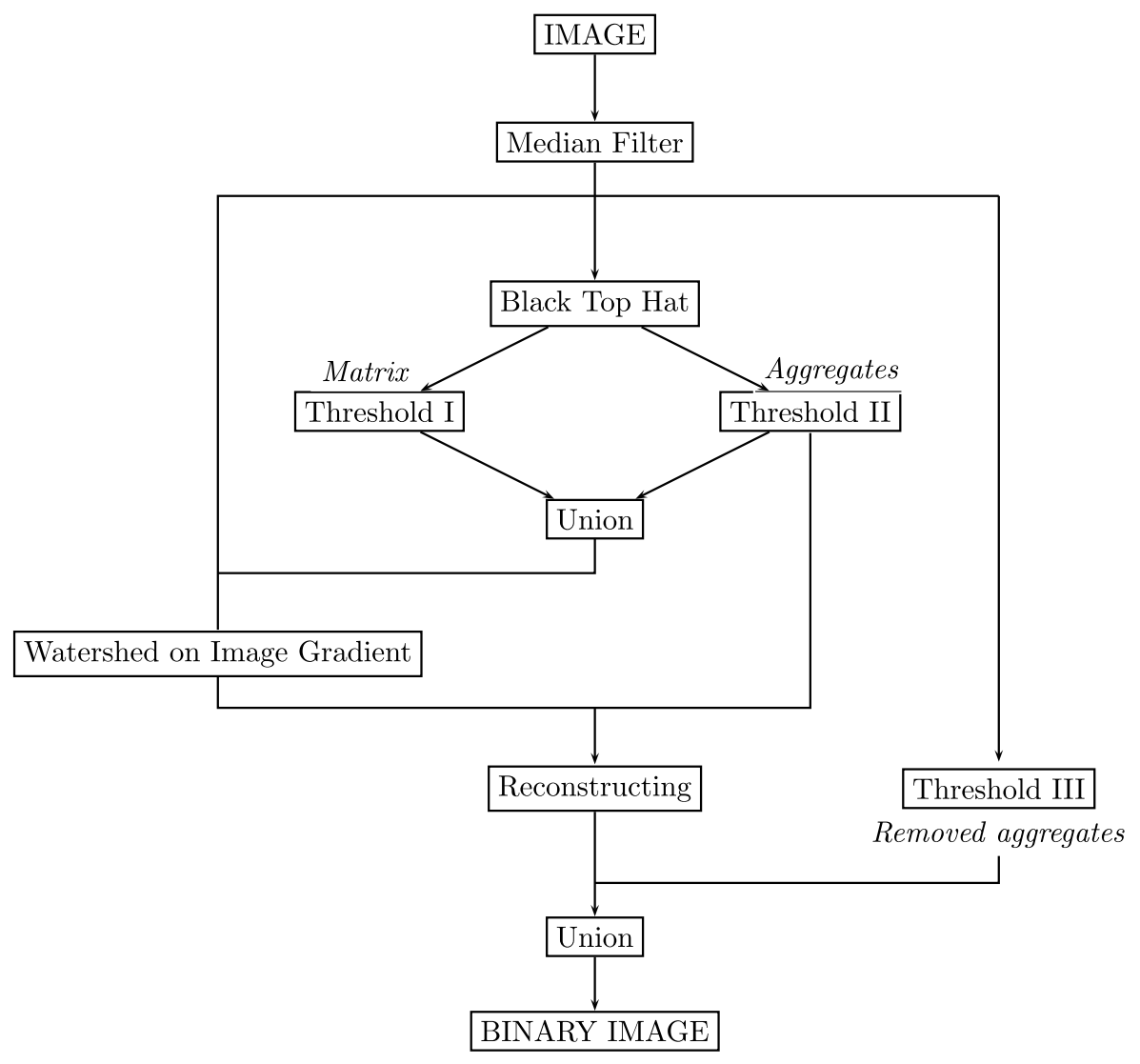

Fig. 3. Algorithm of the segmentation procedure.

In the next section, the distribution of the carbon black aggregates of the binary TEM images is characterized using a statistical approach.

\section{Statistical moments and morphological data}

This section introduces the basic elements retained in this work to characterize the statistical distribution of aggregates. Let $A$ be a set whose distribution is studied and let consider a test set which allows to analyse the statistical distribution of $A$. Here the test set is a two-point set, a three-point set or a disc for the covariance, the third-order moment and the closing curve, respectively.

Covariance. The covariance or two-points probability, (Matheron, 1967) $C(x, x+h)$ of the random set $\mathrm{A}$ is defined by the probability for the two points $x$ and $x+h$ to belong to $A$

$$
C(x, x+h)=P\{x \in A, x+h \in A\} .
$$

When considering a stationary random set $A$, the covariance does not depend on $x$, and is noted $C(h)$.

The covariance depends on the orientation and on the modulus of vector $h$. For a vanishing distance $\|h\|$ separating the two points, the covariance is equal to the one-point probability to belong to the set $A$. This probability is the volume fraction of the random set $A$ in the binary image. In the case of a stationary random set, for an infinite distance $\|h\|$, the two events become independent and thus the covariance is the product of each one-point probability $P\{x \in A\} \times P\{x+$ $h \in A\}$. This asymptotic expression corresponds to the square of the volume fraction of the random set $A$ in the binary image. The value of the distance $\|h\|$ when this sill is reached is the range, or characteristic length of the microstructure. For an isotropic microstructure, the covariance does not depend on the orientation of $h$.

Third-order moment. The third-order moment or three-point probability, $T\left(x, x+h_{1}, x+h_{2}\right)$ of the random set A is defined by the probability for the three points $x, x+h_{1}$ and $x+h_{2}$ to belong to $A$. For the stationary case, it does not depend on $x$, and is defined by

$$
T\left(h_{1}, h_{2}\right)=P\left\{x, x+h_{1}, x+h_{2} \in A\right\} .
$$

In this case, the three points are the vertices of an equilateral triangle, and therefore the moduli of $h_{1}$ and $h_{2}$ are equal to $\|h\|$. Such as in the case of the covariance, for a null distance $\|h\|$, the third-order moment corresponds to the one-point probability. For an infinite distance $\|h\|$, the third-order moment reach 
(a)

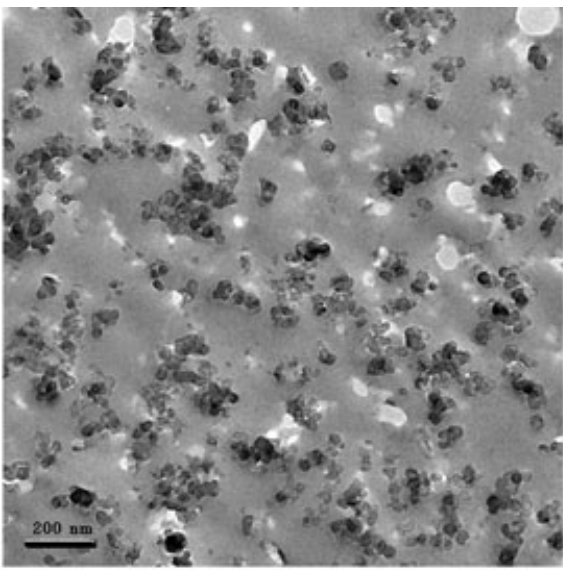

(c)
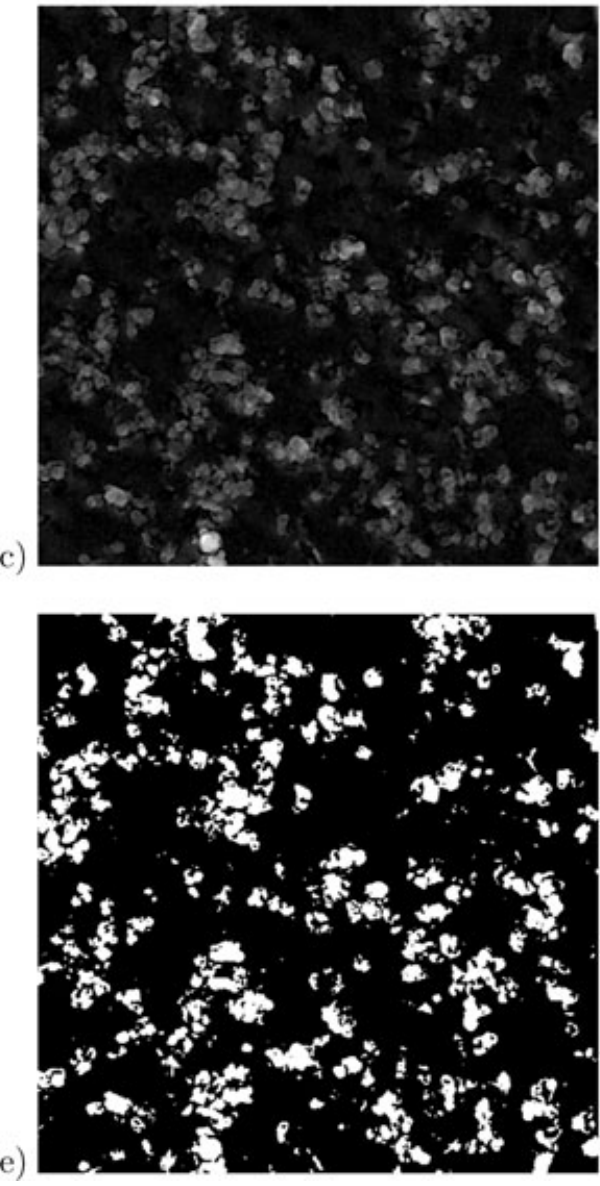
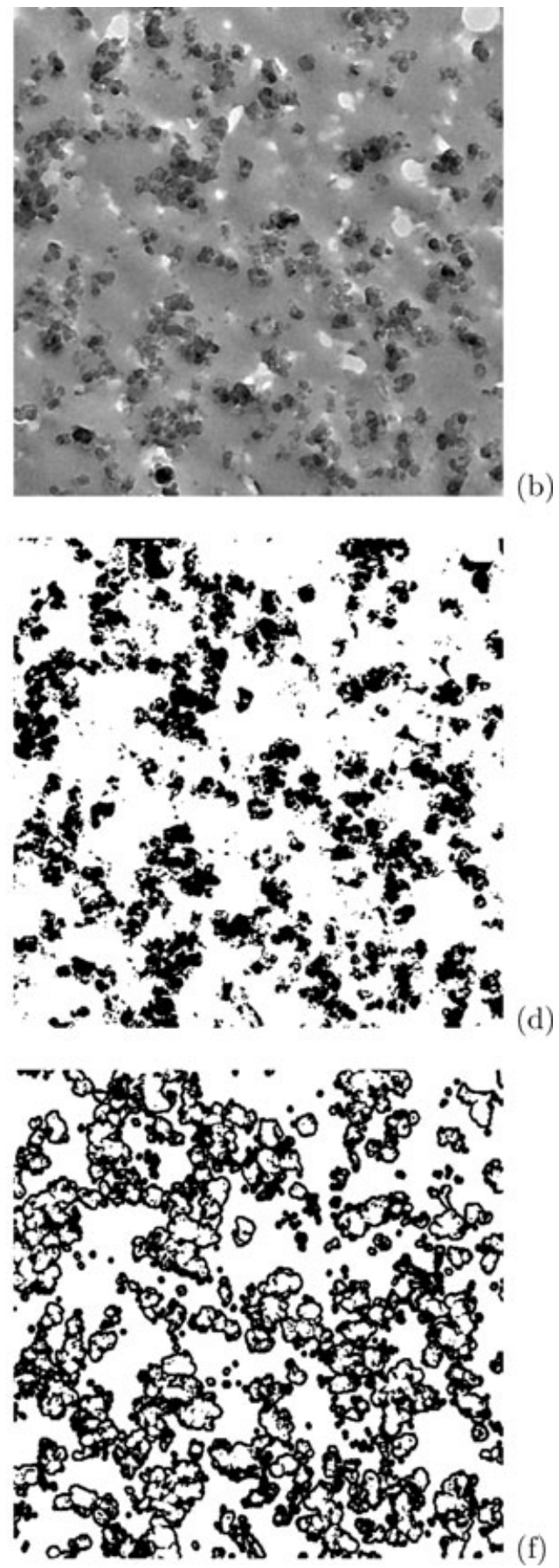

Fig. 4. Segmentation of a TEM micrograph. (a) Initial grey-level image, (b) median filter using a square of three pixels of length, (c) black top hat by a disc with a radius that is equal to 12 pixels, (d) thresholding, (e) thresholding, and (f) union of binary images (d) and (e).

an asymptotic value which is the cube of the volume fraction of the set $A$. The value of the distance $\|h\|$ when the sill is reached, is also a characteristic length of the set. The mean value of this characteristic length over a sample of images, must be equal to the one obtained for the covariance, when using a representative sample of the microstructure in the case of isotropy.
Closing curve. The closing operation of the set $A$ by the set $D$ consists in dilating the set $A$ by $D$ and then in eroding the resulting set by $D$ (Serra, 1982). This operation adds to $A$ some points of the complementary set $A^{\mathrm{c}}$ of $A$, according to a size criterion. The closing curve is defined by the probability for a point to belong to the set $A$ closed by a disc $D(d)$ with an increasing diameter $d$. For a stationary random set, it does not 
(a)
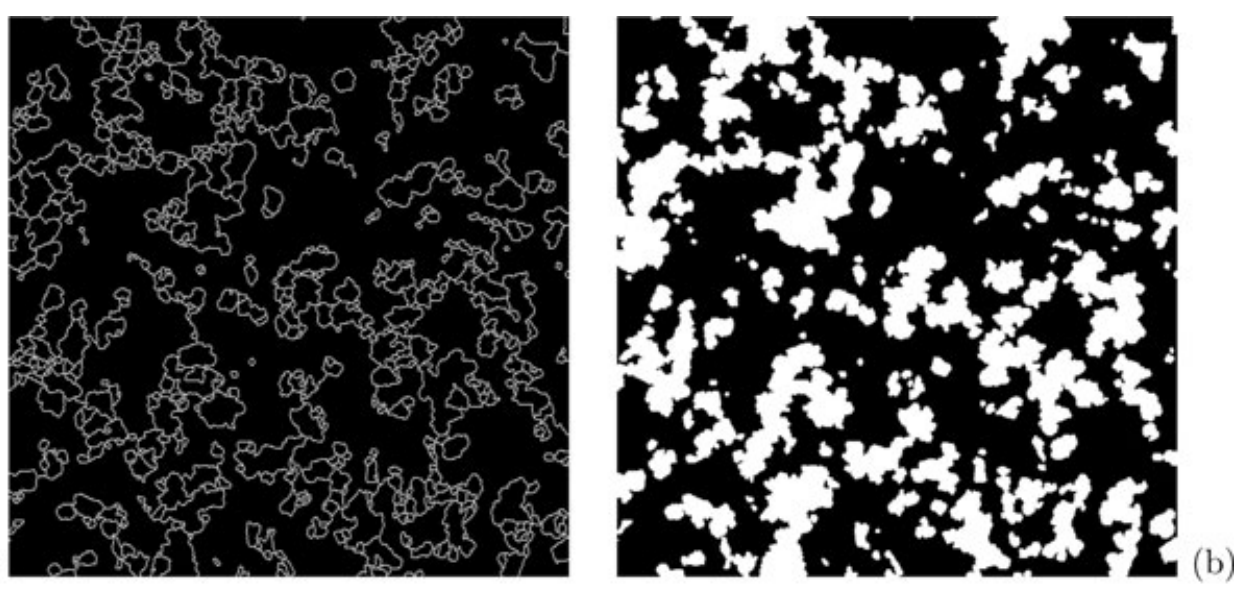

(c)
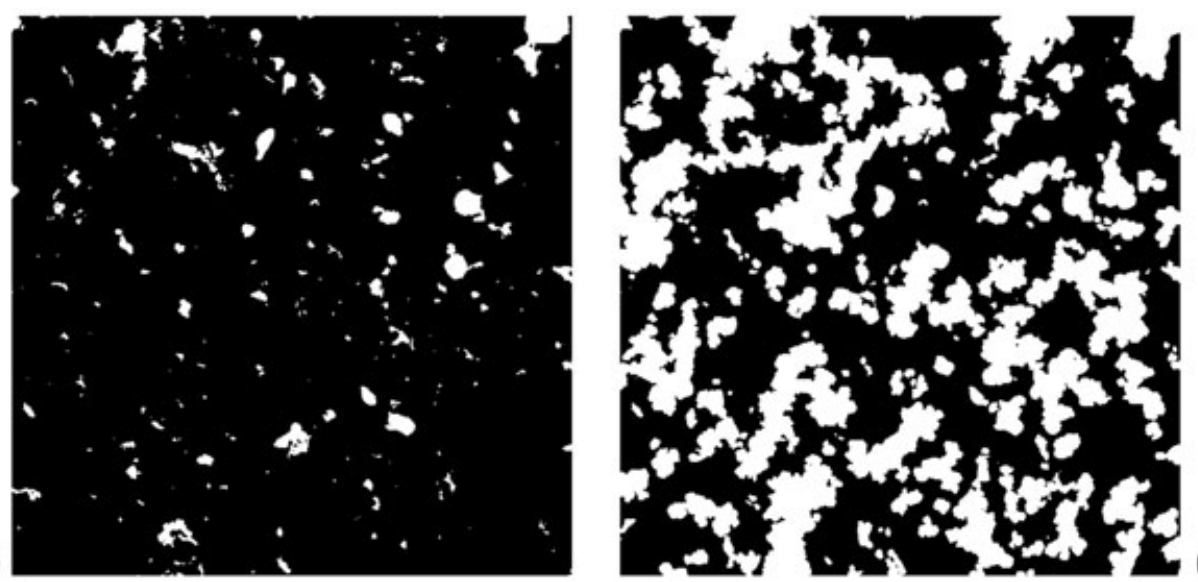

Fig. 5. Segmentation of a micrograph. (a) Watershed on the gradient of the image 4(f), (b) binary image of the visible carbon black particles, (c) thresholding, $S=185$, to extract the prints of erased carbon black particles and (d) final binary image of carbon black.

depend on the location of point $x$. We have

$$
C l(d)=P\{x \in(A \oplus D(d)) \ominus D(d)\} .
$$

Results. Figures 8 and 9 show the covariances and the thirdorder moments measured on images of a representative sample of the material $M_{1}$. Each image is made on a separate slice generated at random in the material. Therefore, a wide range of orientations is sampled in the material. No significant difference appears in the various curves of Figs 8, 9, 11 and 12 , meaning that the microstructure is isotropic at the scale of observation. Additional measurements of covariance were made on two orthogonal directions (the horizontal and vertical orientations in images like in Figs 6 and 7), showing no significant difference either. We can conclude that the distribution of carbon black aggregates is isotropic, which was expected according to the mixing process. Figure 10 shows the corresponding mean covariance and mean thirdorder moment. For both moments, the characteristic length is around 40 pixels hence $128 \mathrm{~nm}$. The value for the null distance $h$ corresponds to the apparent area fraction of carbon black fillers on binary TEM micrographs, around $28 \%$. This value is equal to the double of volume fraction of fillers in material, because of the effect of the thickness of the slice. The area fraction on a real section (with zero thickness) would provide the effective volume fraction of the material (14\%). Figures 11 and 12 show the covariances and the third-order moments of a representative sample of the material $M_{2}$. Figure 13 shows the corresponding mean covariance and mean third-order moment. For both, the characteristic length is around 30 pixels hence $96 \mathrm{~nm}$.

The curves in Figs 8-12 show the fluctuations, that result from the heterogeneity of the microstructure. The variance of the area fraction enables us to provide error bars corresponding to the $95 \%$ interval of confidence. The area fraction range is $24-33 \%$ for material $M_{1}$ and $27-30 \%$ for material $M_{2}$. Figure 14 shows the closing curve for both materials as a function of the diameter $d$ of the disc for the closing operation. The closing curve enables us to describe the matrix morphology. Here, in the case of material $M_{1}$, the proportion of the white phase tends to $100 \%$ for larger sizes, 
(a)

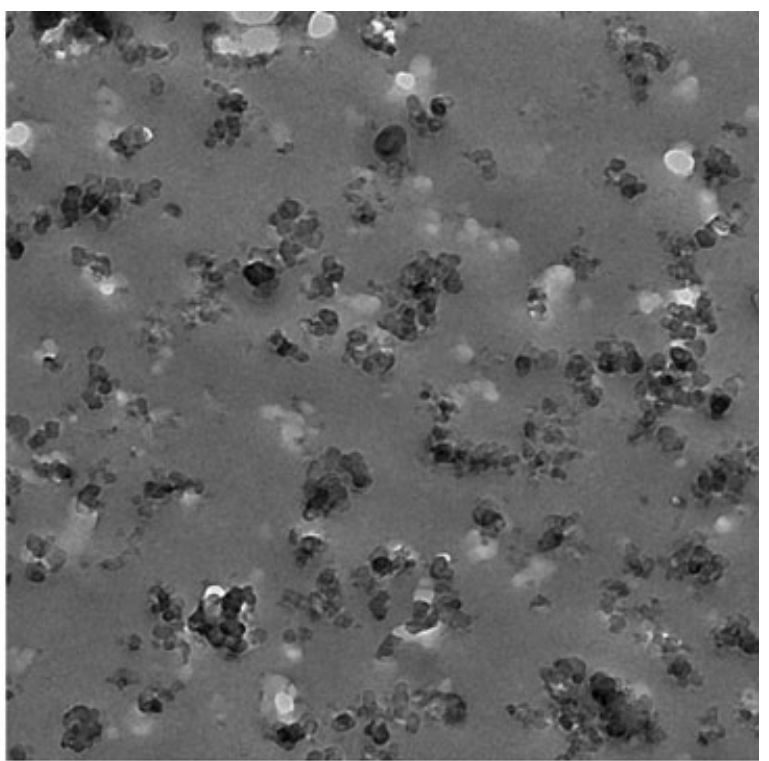

(b)

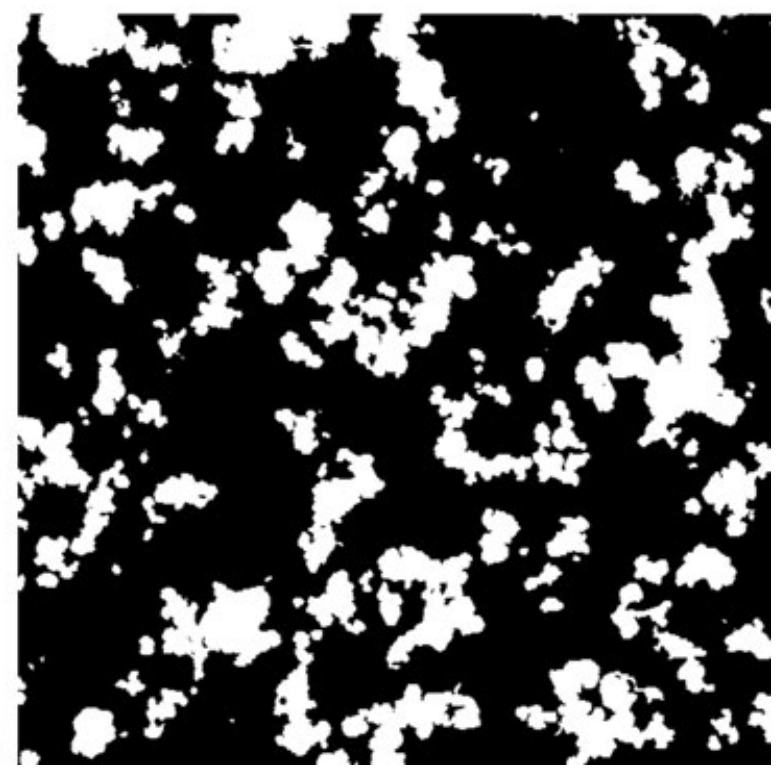

Fig. 6. TEM micrograph of material $M_{1}$ (a) and its binary image (b).

as compared to the material $M_{2}$. Indeed, the material $M_{1}$ is less homogeneous, thus the areas of matrix located between aggregates are larger.

\section{Multi-scale model of microstructure}

This section aims at modelling the morphology of carbon black network in the rubber matrix. The first part introduces the basic primary model which is the Boolean model of spheres. In the second part, this primary model is used to generate the multiscale model that takes the three scales of the microstructure into account.

The Boolean model of spheres. The Boolean model of spheres (Matheron, 1967; Serra, 1982) is obtained by implantation of (a)

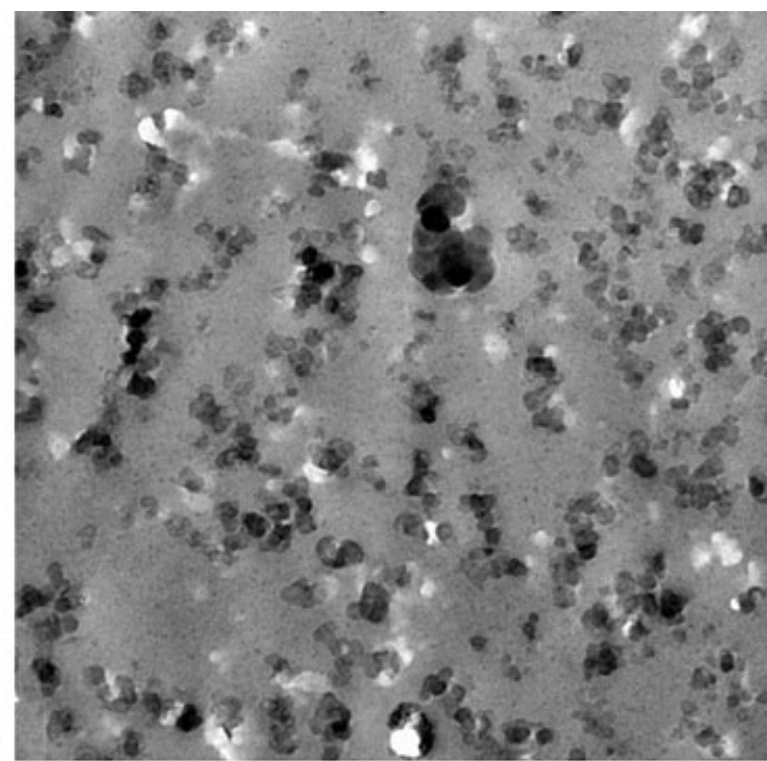

(b)

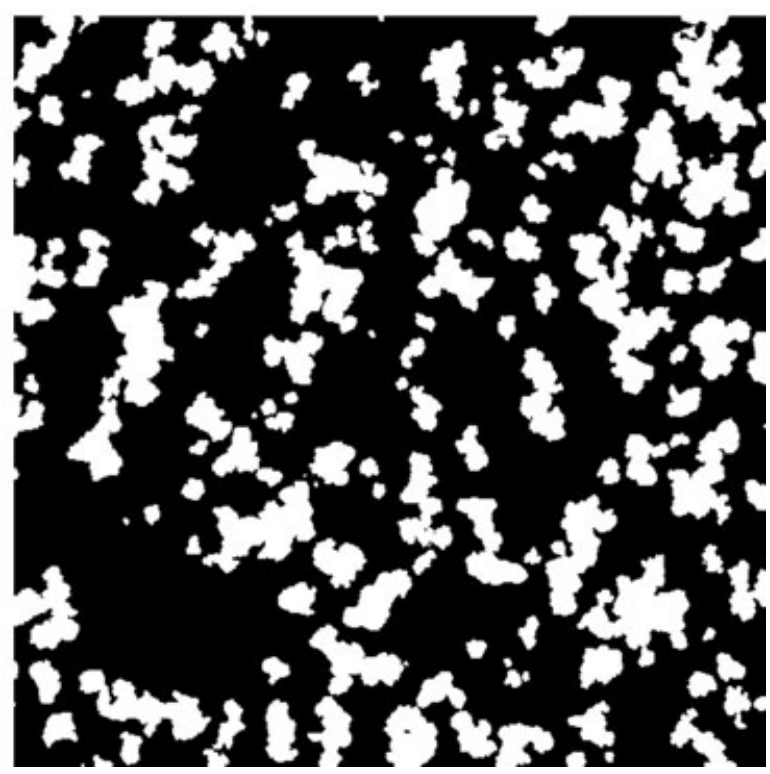

Fig. 7. TEM micrograph of material $M_{2}$ (a) and its binary image (b).

primary spheres $A_{i}^{*}$ on Poisson points $x_{i}$, with possible overlaps $\left(A=\cup A_{i}^{*}\right)$. The covariance $Q(h)$ of the complementary set $A^{\mathrm{c}}$, can be expressed as follows:

$$
\begin{aligned}
Q(h)= & \left(1-V v_{A}\right)^{2} \exp (\theta K(h)), \\
= & \left(1-V v_{A}\right)^{2-r(h)},
\end{aligned}
$$

with

$$
\begin{gathered}
r(h)=\frac{K(h)}{K(0)}, \\
K(h)=\bar{V}\left(A^{*} \cap A_{-h}^{*}\right),
\end{gathered}
$$

where $V v_{A}$ is the volume fraction of the set $A, \theta$ is the Poisson point process intensity, $V$ is the volume and $K(h)$ and $r(h)$ 


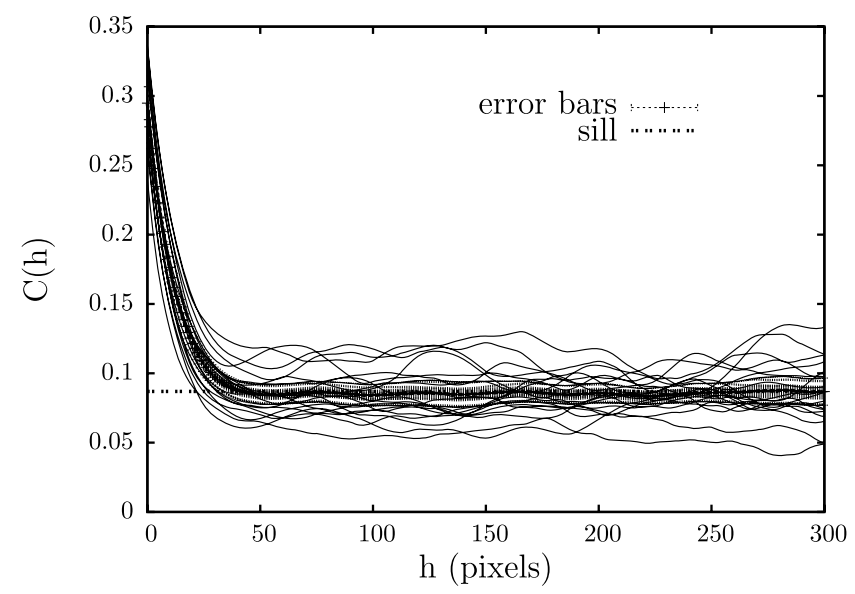

Fig. 8. The covariances of the images of material $M_{1}$.

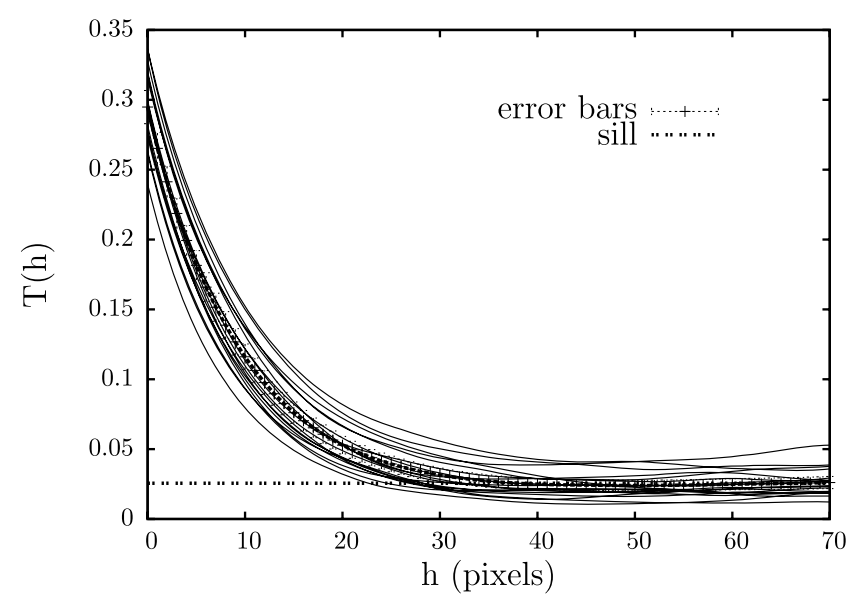

Fig. 9. The third-order moments of the images of material $M_{1}$.

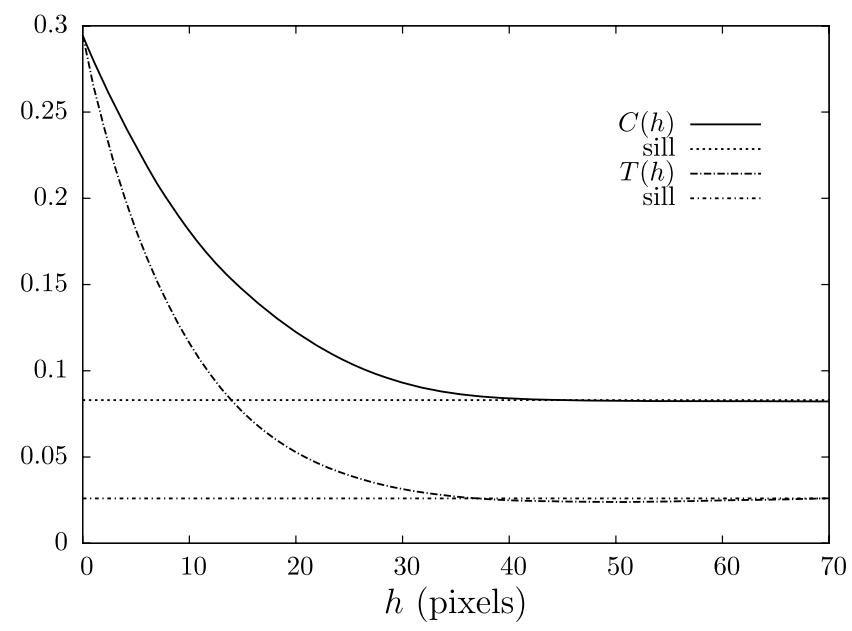

Fig. 10. The mean covariance and the mean third-order moment of material $M_{1}$.

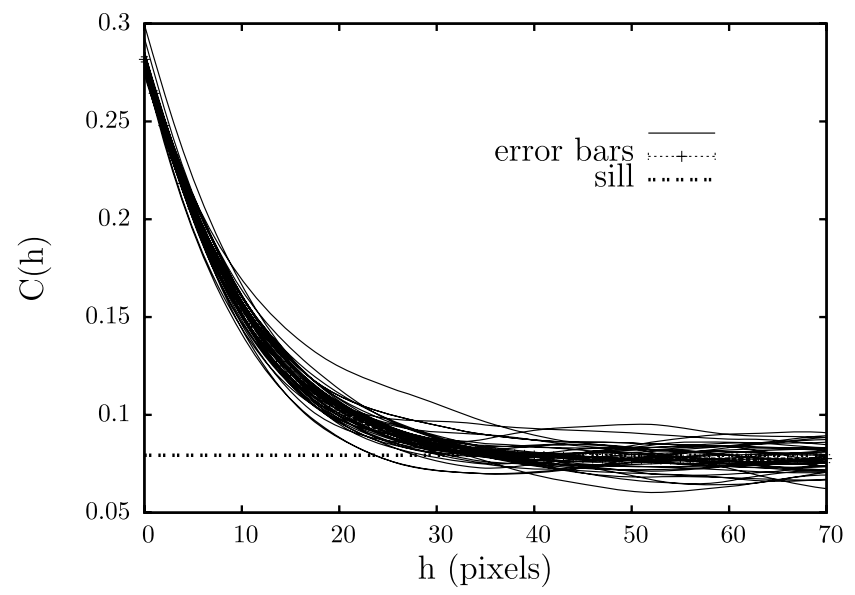

Fig. 11. The covariances of the images of material $M_{2}$.

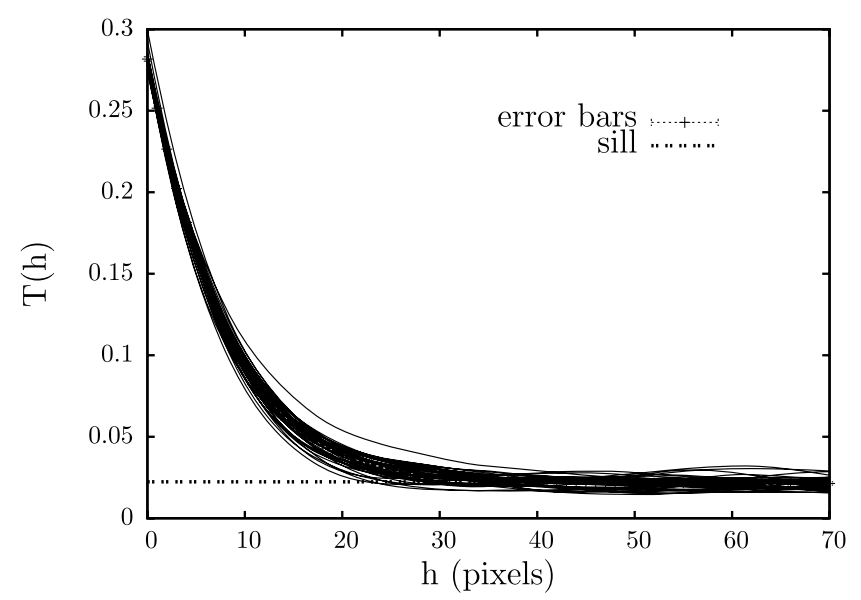

Fig. 12. The third-order moments of the images of the material $M_{2}$.

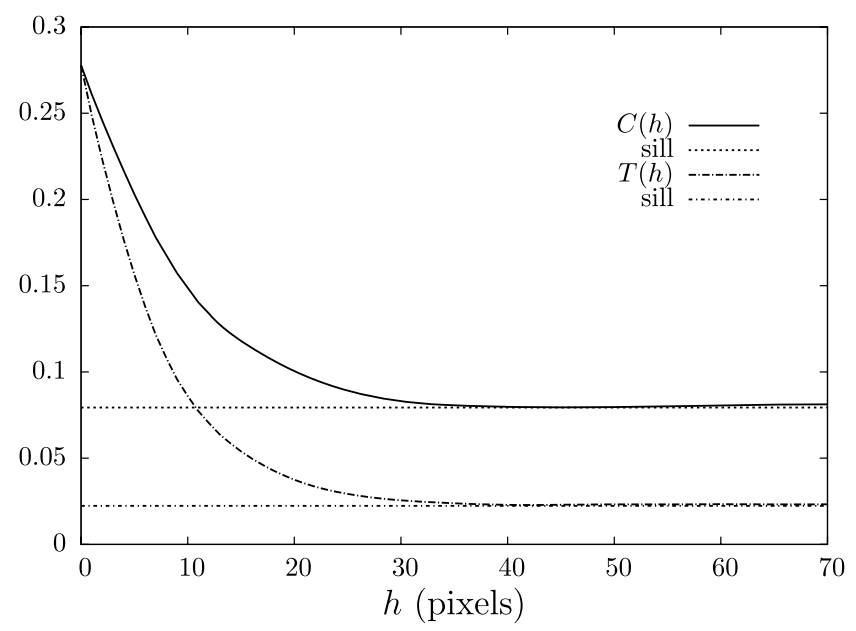

Fig. 13. The mean covariance and the mean third-order moment of material $M_{2}$. 


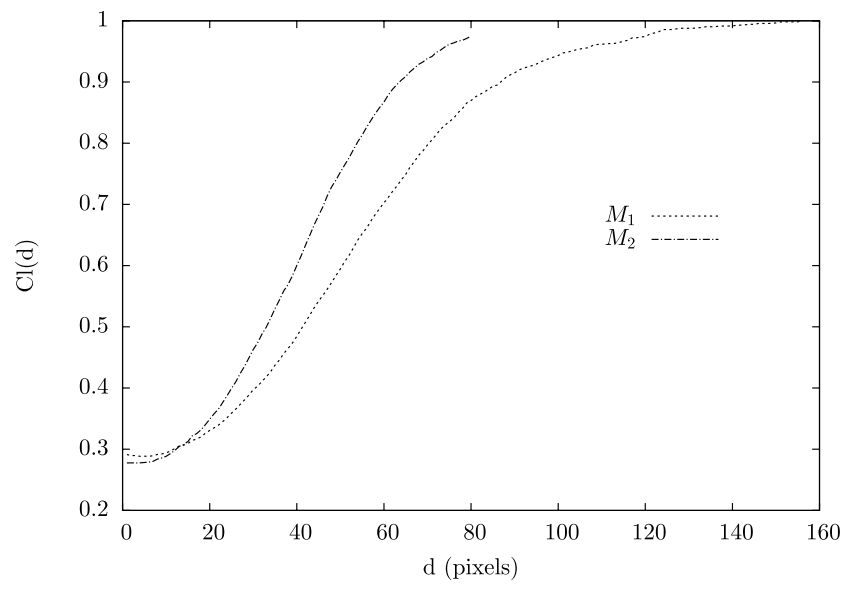

Fig. 14. The mean closing curves of materials $M_{1}$ and $M_{2}$.

the geometrical covariogram of the grains and the normalized one, respectively. The covariance of a Boolean model of spheres depends on the volume fraction, $V v_{A}$ and on the radius of the spheres, $R_{A}$, which can be constant or can follow a statistical distribution.

A combination of three Boolean Models. To model the multiscale microstructure of rubber with carbon black fillers, a combination of three basic Boolean models is needed (Jeulin, 1991) as in the case of carbon black in a polymeric matrix (Jeulin \& Le Coënt, 1995; Savary et al., 1999; Delarue, 2001; Moreaud \& Jeulin, 2005; Jean et al., 2007). Each Boolean model defines a physical scale of the microstructure identified on TEM images: the particles, the aggregates and the matrix.

For the carbon black particles, a Boolean model of spheres with a radius following a Gaussian distribution is considered (Fig. 23a). The corresponding set is called $A_{p}$. According to the nature of the carbon black, the Gaussian law is troncated at 10 and $30 \mathrm{~nm}$ and has a mean value equal to $20 \mathrm{~nm}$. According to the Boolean model, the particles can overlap in the final aggregates. This feature is retrieved physically. Indeed, carbon black particles exhibit a turbostratic structure of graphite planes (Fig. 15a) as illustrated in Fig. 15(b) with the model presented in (Donnet, 2003). The turbostratic structure is the key feature that enables the particles to merge and to create aggregates.

For the aggregates, some measures of the shape factor were performed on TEM observations for about thousands aggregates by the partner, Michelin. From the TEM observations, the following morphological measurements were done on projection on a plane of the initial threedimensional aggregates. Figure 16 shows the proportion of aggregates as a function of the shape factor measured on the convex hull of the projections. The values of shape factor are in a range 0.5-1.2 and the mean value is equal to 0.8 . Thus, most of aggregates do not correspond to spheres but more to ellipsoids, with an elliptic projection. Here, due to

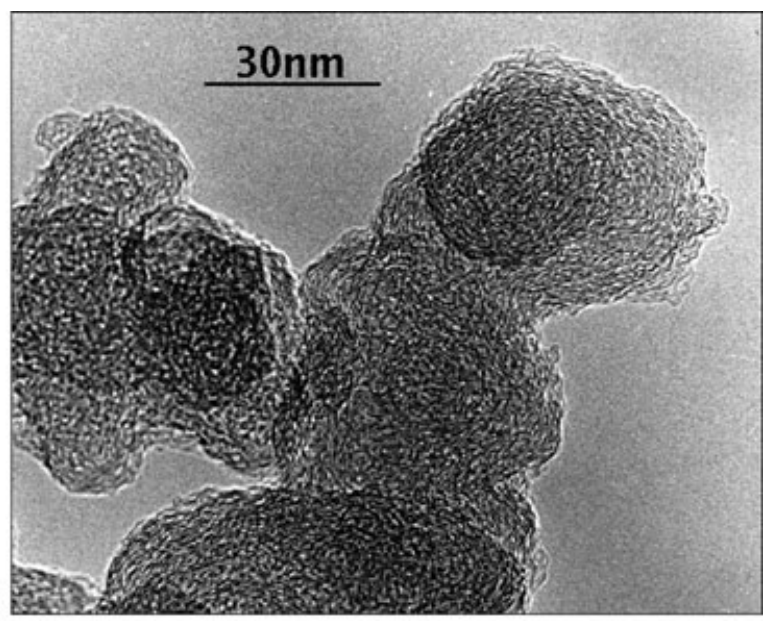

(b)

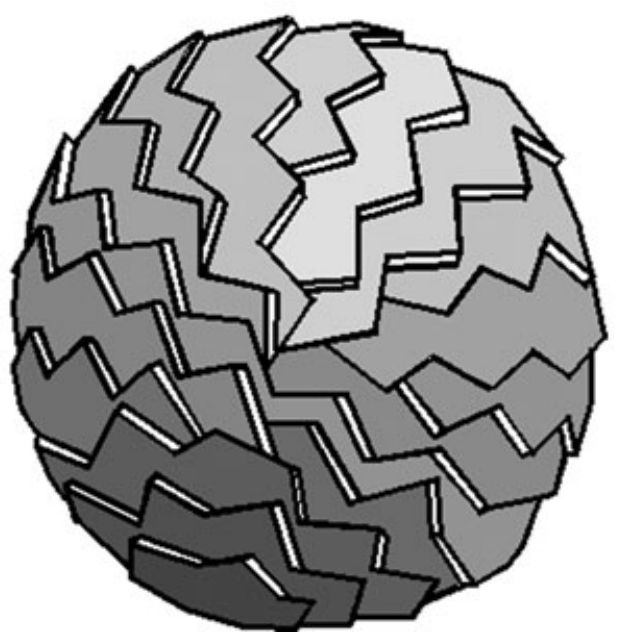

Fig. 15. Structure of carbon black particle : (a) a TEM observation of the turbostratic structure of a particle and (b) a model of the structure, after (Donnet, 2003).

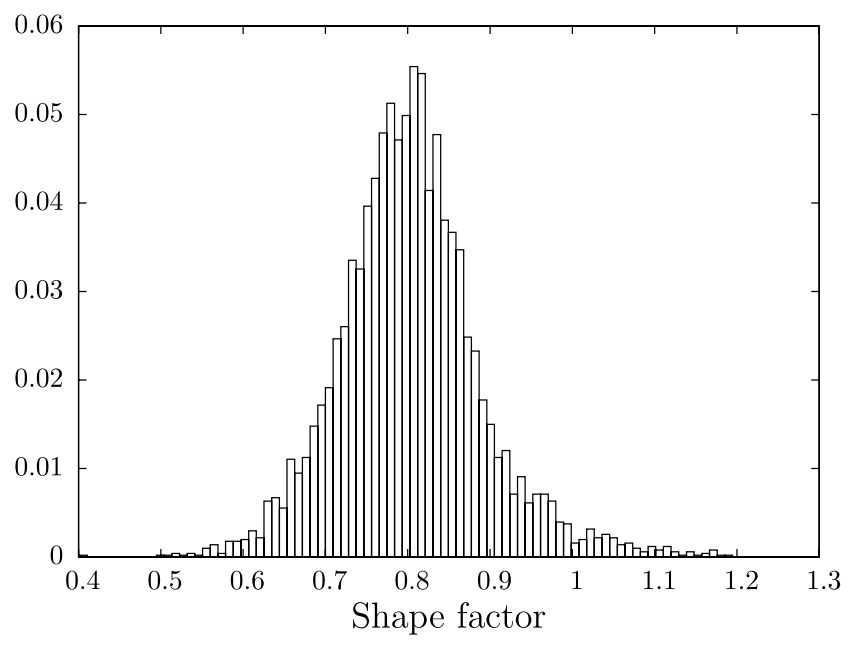

Fig. 16. Distribution of shape factor measured on the convex hull of the projection of about 1000 aggregates (Michelin). 


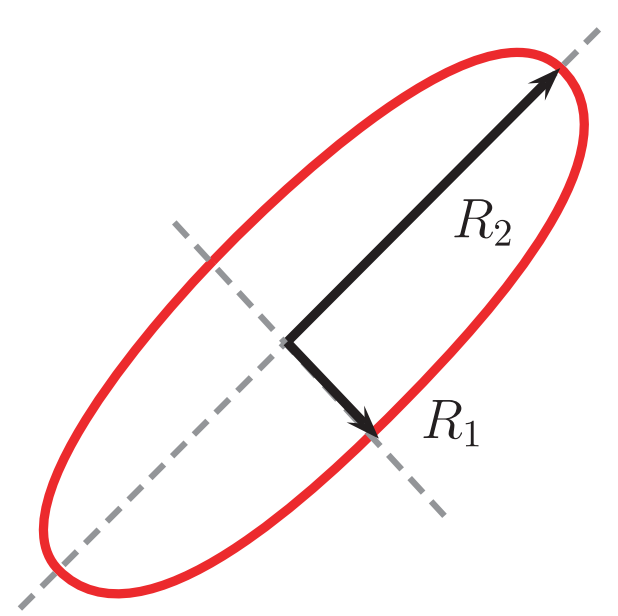

Fig. 17. Ellipse with its two characteristic lengths $R_{1}$ and $R_{2}$.

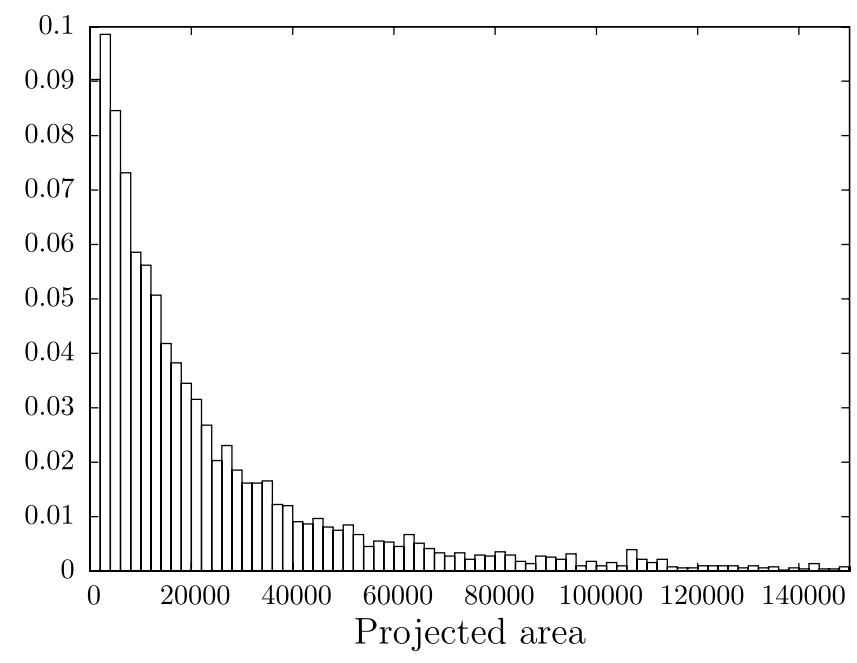

Fig. 18. Distribution of the projected area measured on about 1000 aggregates (Michelin).

the two-dimensional analysis, the assumption of spheroidal aggregates, with two characteristic lengths, named $R_{1}$ and $R_{2}$, is retained (Fig. 17).

The shape factor $F F$ is given as a function of perimeter $P$ and area $A$ by

$$
F F=\frac{4 \pi \times A}{P^{2}}
$$

Figures 18 and 19 show the distribution of area and perimeter of projections for the thousands aggregates, respectively. In the case of ellipses, the area and the perimeter are expressed as follows, as functions of the two characteristic lengths $R_{1}$ and $R_{2}$

$$
\left\{\begin{array}{l}
A=\pi R_{1} R_{2}, \\
P \simeq 2 \pi \sqrt{\frac{1}{2}\left(R_{1}^{2}+R_{2}^{2}\right)} .
\end{array}\right.
$$

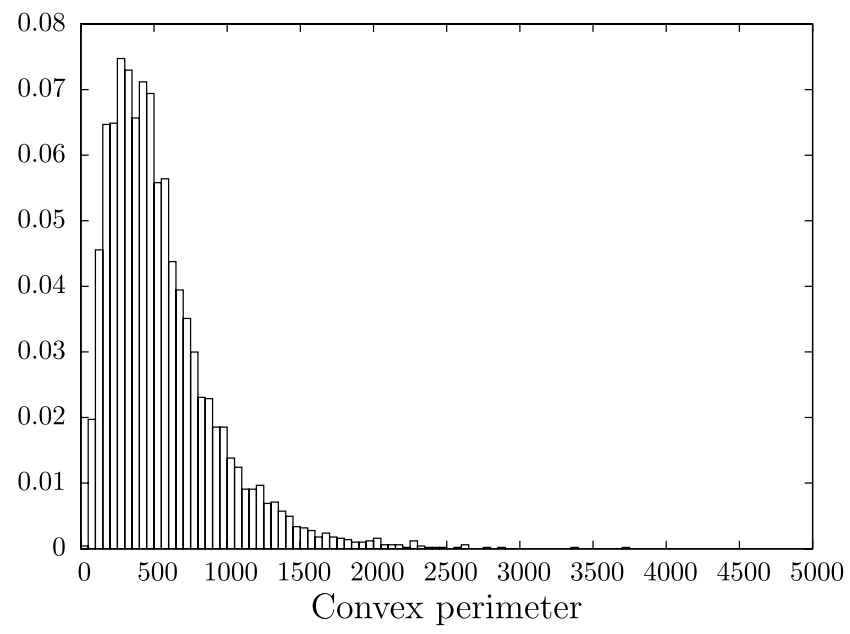

Fig. 19. Distribution of the perimeter of projections measured about thousands aggregates (Michelin).

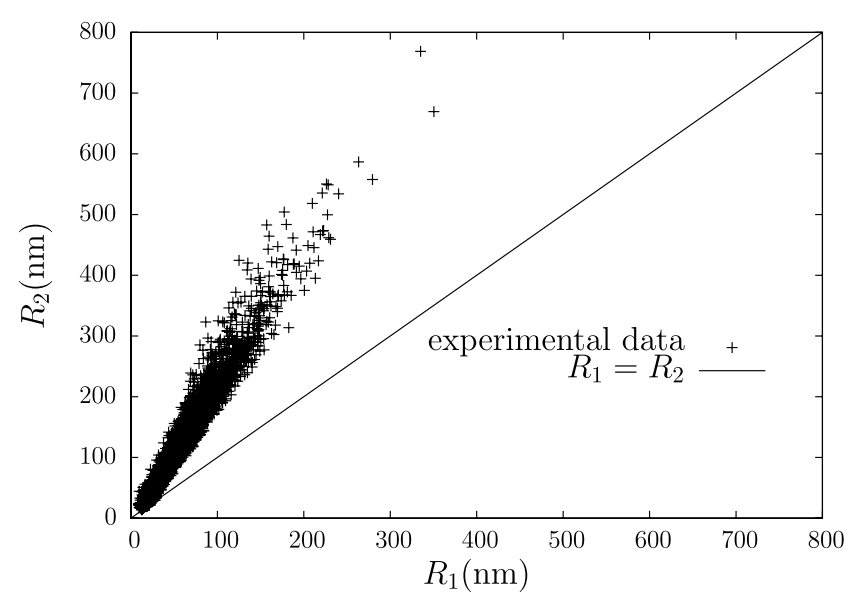

Fig. 20. Obtained couples $\left(R_{1}, R_{2}\right)$.

$R_{1}$ is written as function of $R_{2}$

$$
R_{1}^{2}=\frac{1}{2}\left(\frac{P}{\pi}\right)^{2}-R_{2}^{2} .
$$

From the square of the Eq. (9)

$$
R_{2}^{4}-\frac{1}{2}\left(\frac{P}{\pi}\right)^{2} \times R_{2}^{2}+\left(\frac{A}{\pi}\right)^{2}=0
$$

Finally, the couple of lengths, $R_{1}$ and $R_{2}$ is obtained as follows:

$$
\left\{\begin{array}{l}
R_{1}^{2}=\frac{1}{4}\left(\frac{P}{\pi}\right)^{2}+\frac{1}{2} \sqrt{\frac{1}{4}\left(\frac{P}{\pi}\right)^{4}-4\left(\frac{A}{\pi}\right)^{2}}, \\
R_{2}^{2}=\frac{1}{4}\left(\frac{P}{\pi}\right)^{2}-\frac{1}{2} \sqrt{\frac{1}{4}\left(\frac{P}{\pi}\right)^{4}-4\left(\frac{A}{\pi}\right)^{2}} .
\end{array}\right.
$$

Figure 20 illustrates the couples $\left(R_{1}, R_{2}\right)$ obtained from the measurement on TEM observations of perimeter and area on the thousands projected aggregates. A linear dependence is found between $R_{1}$ and $R_{2}, R_{2}=2.5535 R_{1}+12.0916$ from a linear regression. Figures 21 and 22 give the distribution of $R_{1}$ 


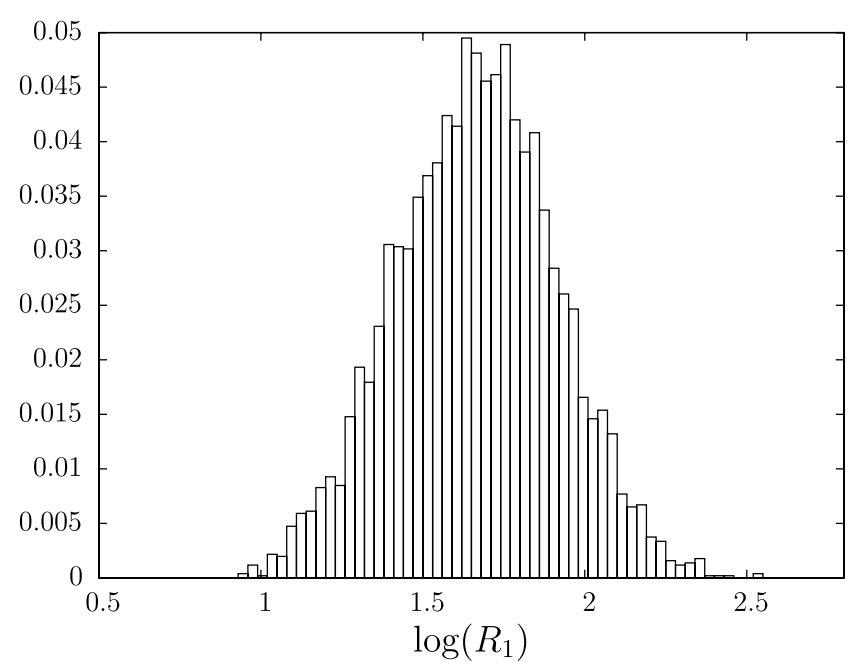

Fig. 21. Log-normal distribution of $R_{1}$.

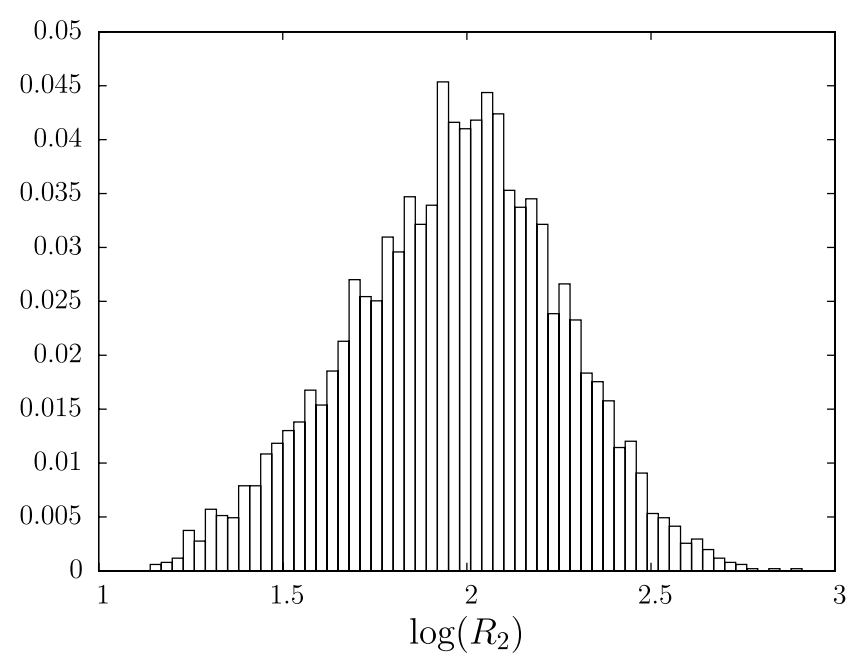

Fig. 22. Log-normal distribution of $R_{2}$.

and $R_{2}$, respectively. These distributions follow a log-normal distribution.

According to these results, the aggregates are modelled by a Boolean model of spheroids with axis length following a log-normal distribution with a mean value equal to $200 \mathrm{~nm}$ (Fig. 23b) and with a uniform orientation. We first generate a realization $R_{1}$. Then $R_{2}$ is estimated from the linear regression. A prolate spheroid is generated with two equal axis $R_{1}(<$ $R_{2}$ ). In the following, these spheroids are called the inclusions because they generate the final aggregates. The resulting set is $A_{i}$. For the matrix, a Boolean model of spheres with constant radius is considered (Fig. 23c). These spheres are called the exclusions because there are not aggregates into these Boolean model. The resulting set is $A_{e}$.

The final multiscale model, defined by the set $A_{C B}$ of carbon black aggregates, is described by the particles whose centres belong to the intersection set of the inclusion $A_{i}$

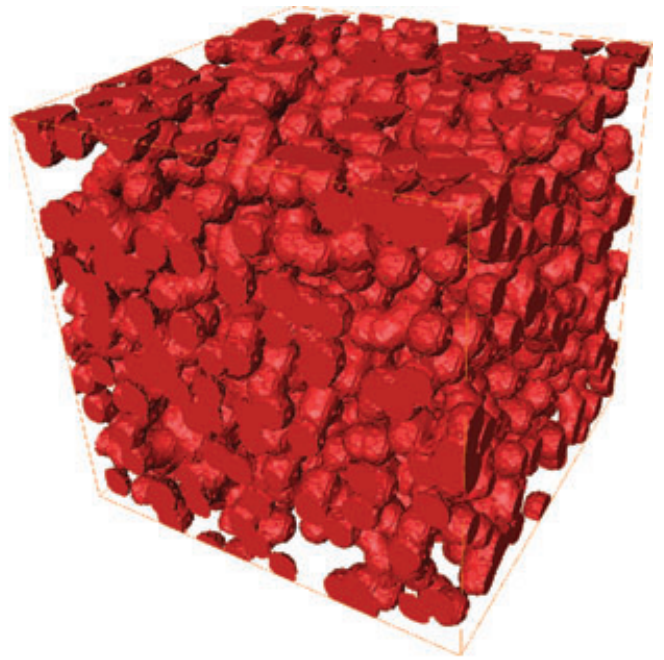

(a)

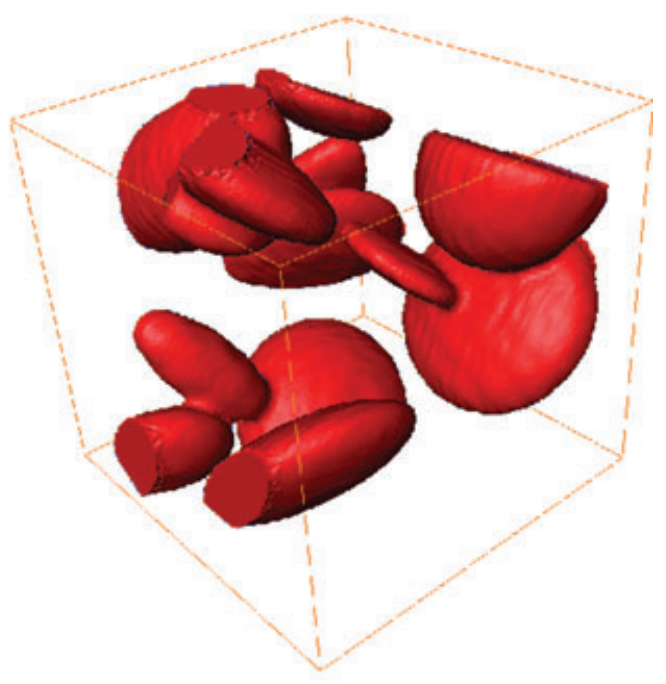

(b)

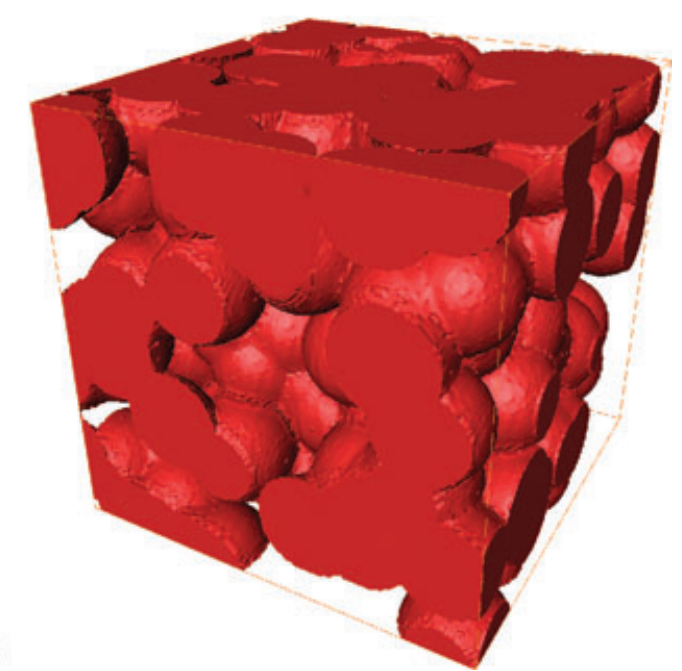

Fig. 23. Multiscale model of microstructure: (a) Boolean model of spherical particles $\left(A_{p}\right)$; (b) Boolean model of spheroidal inclusions $\left(A_{i}\right)$ and (c) Boolean model of spherical exclusions $\left(A_{e}\right)$. 
(a)

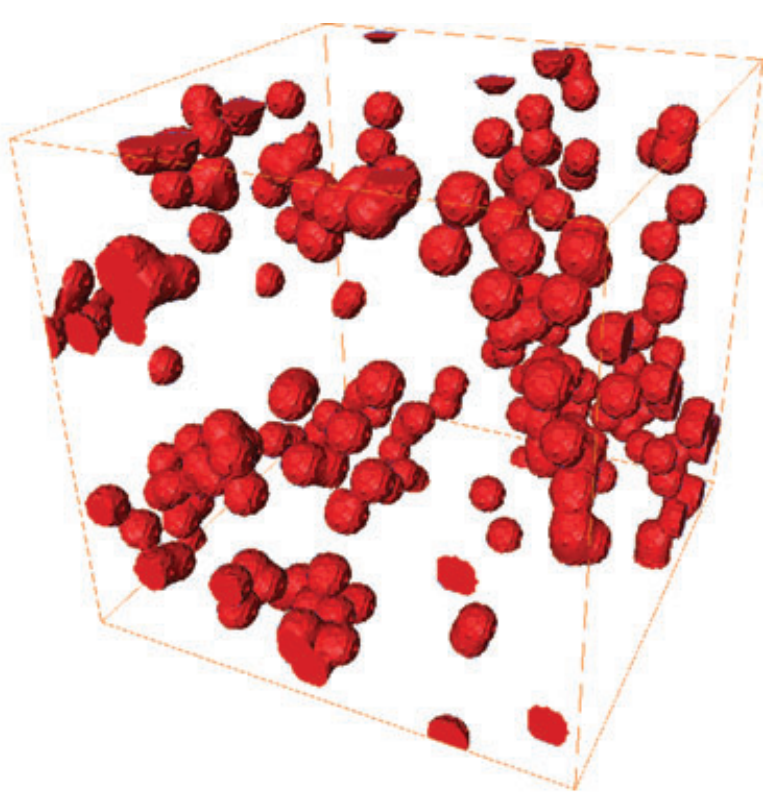

(b)

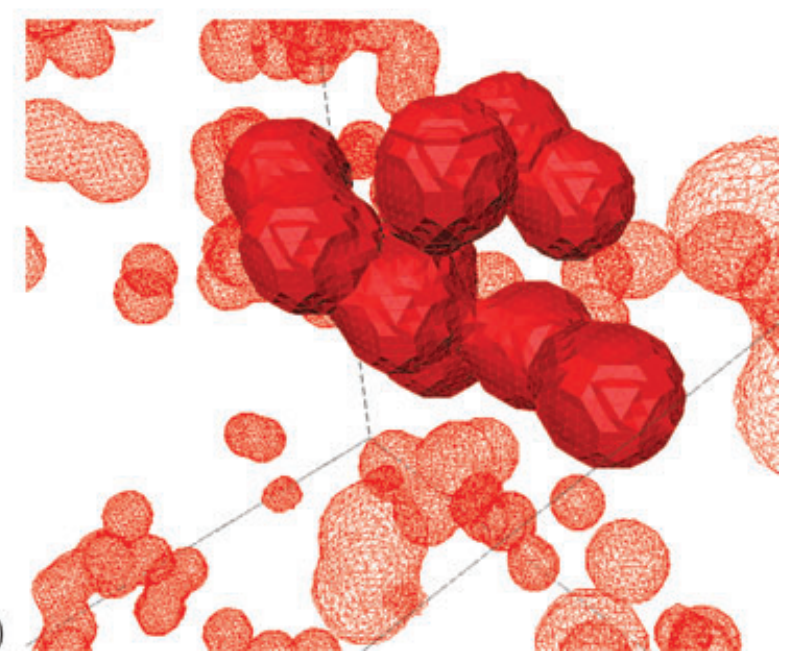

Fig. 24. (a) Final multiscale model of microstructure and (b) an example of carbon black aggregate in the simulated microstructure.

with the complementary set of exclusions $A_{e}^{\mathrm{c}}$ (Fig. 24a). Figure 24(b) illustrates the structure of an aggregate in the simulated microstructure. This model is a Boolean Cox model where the usual Poisson point process of the centres of the standard Boolean model is replaced by a Poisson point process limited to $A_{i} \cap A_{e}^{\mathrm{c}}$. In this model, four parameters are unknown, the volume fraction of each scale, $V v_{p}, V v_{i}$ and $V v_{e}$ and the radius of the exclusion spheres $R_{e}$. To model the microstructure of the material, these four parameters must be estimated from statistical moments and morphological data measured on TEM images. Because no closed-form analytical expression is available for the corresponding statistical data, the identification will be made in the next section from microstructure simulations.

\section{Parameter identification method}

This subsection describes the original method proposed in this work to identify the multi-scale microstructure of rubber with carbon black aggregates. This method can be easily extended to the case of any kind of microstructure. First, the two steps of the algorithm are introduced. Then, the results obtained in the case of the two materials $M_{1}$ and $M_{2}$ are presented.

The algorithm follows two steps. The first step consists in finding a primary set of parameters adjusting the analytic expression of the covariance on the experimental data. This analytic expression comes from a model of microstructure close to the described Boolean Cox model, but slightly different, for which a closed form of the covariance is available. This model is the intersection of three Boolean models of spheres with a constant radius for each one. The resulting set is written as follows:

$$
A_{C B}=A_{p} \cap A_{i} \cap A_{e}^{c} .
$$

Thus, the covariance of the carbon black aggregates of the model reads

$$
\begin{aligned}
C_{C B}(h)= & C_{p}(h) \times C_{i}(h) \times Q_{e}(h) \quad \text { with } \\
& \times\left\{\begin{array}{l}
C_{p}(h)=Q_{p}(h)+1-2\left(1-V v_{p}\right), \\
C_{i}(h)=Q_{i}(h)+1-2\left(1-V v_{i}\right), \\
Q_{e}(h)=\left(1-V v_{e}\right)^{2-r_{e}(h)} .
\end{array}\right.
\end{aligned}
$$

The thickness $t$ of the TEM slice ( $t \simeq 40 \mathrm{~nm}$ ) is similar to the size of a particle ( $R_{p} \simeq 20 \mathrm{~nm}$ ). Thus, up to two particles can be seen on the TEM images, in the thickness. To take this thickness into account, the analytic expression of the resulting covariance has to be corrected by modifying the covariance that corresponds to the particle set (Savary et al., 1999):

$$
C_{C B \oplus t}(h) \simeq C_{p \oplus t}(h) \times C_{i}(h) \times Q_{e}(h) .
$$

In the expression (5), the spheres of carbon black nanoparticles become sphero-cylinders, as a result of the projection through the thickness. These sphero-cylinders are obtained by dilating the spheres by a vector whose norm corresponds to the thickness of sections. Here, the effect of the dilatation on the larger spheres of the model corresponding to aggregates and to zones of exclusions is neglected. Thus, the apparent volume fraction is written as follows, using the theoretical expression given for the Boolean model:

$$
\left(1-V v_{p}^{\text {app }}\right) \simeq P\left\{x \in A^{\mathrm{c}} \oplus t\right\}=\left(1-V v_{p}\right)^{\frac{\bar{V}\left(A^{*} \oplus t\right)}{\bar{V}\left(A^{*}\right)}}
$$

and the geometrical covariogram for a vector $h$ parallel to the projection plane reads

$$
K_{A_{p \oplus t}^{*}}(h)=K_{S}(h)+t K_{D}(h),
$$

where, $K_{S}(h)$ is the geometrical covariogram of a sphere and $K_{D}(h)$ the one of a disc with a constant radius. Indeed, a 
sphero-cylinder can be described as the union of a cylinder with two hemispheres located at the ends. For this first part of the algorithm, the spheroidal shapes to generate the aggregates are considered as spheres associated with a constant radius. Similarly, the radius of spherical-shaped particles is constant. Finally, six parameters are fitted from the analytic covariance: the three volume fractions and the radius of each Boolean model. In the obtained set of parameters, only the four initial unknowns are then introduced as an initialized set in the optimization part of the algorithm.

In the second step of the algorithm, the four parameters, $V v_{p}, V v_{i}, V v_{e}$ and $R_{e}$ are estimated by minimizing the error on the covariance, on the third-order moment and on the closing curve. This optimization consists in computing virtual TEM images from three-dimensional simulated microstructures and in computing a least square error between the corresponding statistical moments and morphological data to the experimental one. To create a numerical TEM image, a slice with the same thickness as the TEM slice is extracted (Fig. 25b) from a three-dimensional simulation of microstructure of $1500 \mathrm{~nm}$ length (Fig. 25a). This numerical slice is composed by two-dimensional images of 1-pixel thickness. These two-dimensional images are projected on a plane to create the equivalent TEM thick section $(40 \mathrm{~nm})$ (Fig. 25c). For a set of parameters, around 40-50 numerical TEM images are generated. The mean statistical moments and closing curve are then measured over the sample.

The optimization procedure resorts to a LevenbergMarquardt algorithm implemented in the Z-Opt module of Zset (or ZeBuLoN in French) (Mines ParisTech, 2003). To obtain relevant values, the parameters are bounded. First of all, all the volume fractions are obviously smaller than $100 \%$. The volume fractions of particles and zones of exclusions are higher than the percolating volume fraction for a Boolean model of spheres that is close to $30 \%$. Indeed, the particles percolate in an aggregate and the matrix percolates in the material. The volume fraction of inclusion has a range 0$100 \%$. The radius of the spheres describing the zones of exclusions has a range $20-200 \mathrm{~nm}$ which corresponds to the mean radius of a particle and to the mean size of an aggregate for the lower and upper bounds, respectively. Using this range, the higher zones of exclusions can be described as the union of smaller spheres. During the optimization, the parameters are incremented according to the LevenbergMarquardt algorithm. The increment is initialized as $10 \%$ of the initial value for all the parameters. This method of identification is original in the sense of optimizing on simulation of microstructures and can be used for any kind of geometry of microstructures and for any sort of observations.

In the next section, the model of microstructure of the two materials of the study is identified based on this calibration method.
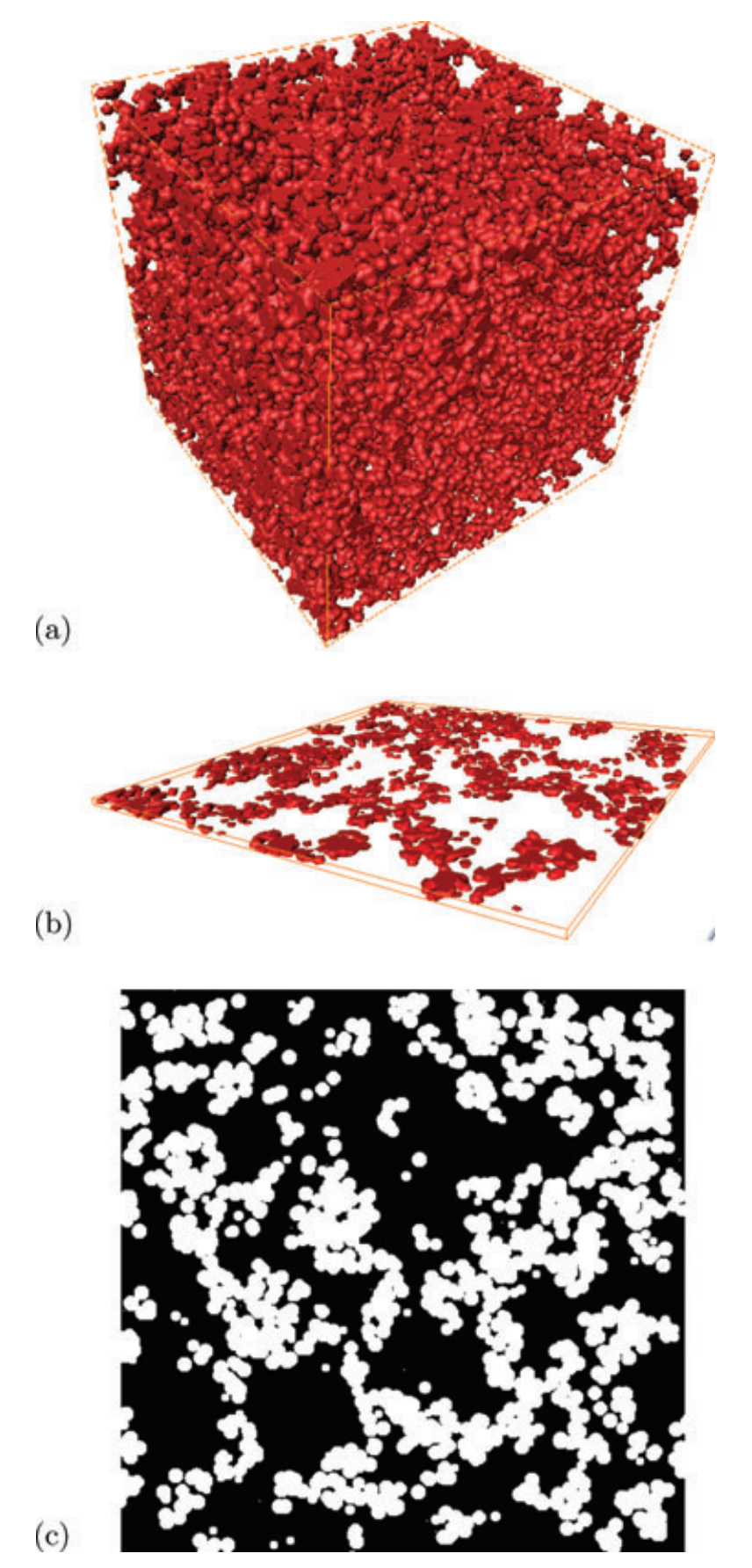

Fig. 25. Simulation of a numerical TEM image from a three-dimensional simulations of the microstructure: (a) three-dimensional simulation of microstructure, (b) perspective view of a thick numerical slice and (c) projected numerical TEM thick slice.

\section{Results}

The identified statistical morphological data are presented in the case of the material $M_{2}$ to illustrate the method. All the final parameters obtained for both materials are given in Tables 1 and 2 . 
Table 1. Set of parameters fitted on the analytic covariance for both materials, $M_{1}$ and $M_{2}$.

\begin{tabular}{ccccccc}
\hline & \multicolumn{2}{c}{ Particles } & \multicolumn{2}{c}{ Inclusions } & \multicolumn{2}{c}{ Exclusions } \\
& $R_{p}(\mathrm{~nm})$ & $V v_{p}^{\text {app }} / V v_{p}$ & $R_{i}(\mathrm{~nm})$ & $V v_{i}$ & $R_{e}(\mathrm{~nm})$ & $V v_{e}$ \\
\hline$M_{1}$ & 15 & $0.72 / 0.35$ & 90 & 0.52 & 62 & 0.22 \\
$M_{2}$ & 13 & $0.93 / 0.44$ & 52 & 0.66 & 60 & 0.55 \\
\hline
\end{tabular}

Table 2. Set of parameters identified on simulated microstructure for both materials, $M_{1}$ and $M_{2}$.

\begin{tabular}{ccccc}
\hline \multicolumn{2}{c}{ Particles } & Inclusions & \multicolumn{2}{c}{ Exclusions } \\
& $V v_{p}$ & $V v_{i}$ & $R_{e}(\mathrm{~nm})$ & $V v_{e}$ \\
\hline$M_{1}$ & 0.5 & 0.5 & 56 & 0.46 \\
$M_{2}$ & 0.5 & 0.47 & 72 & 0.41 \\
\hline
\end{tabular}

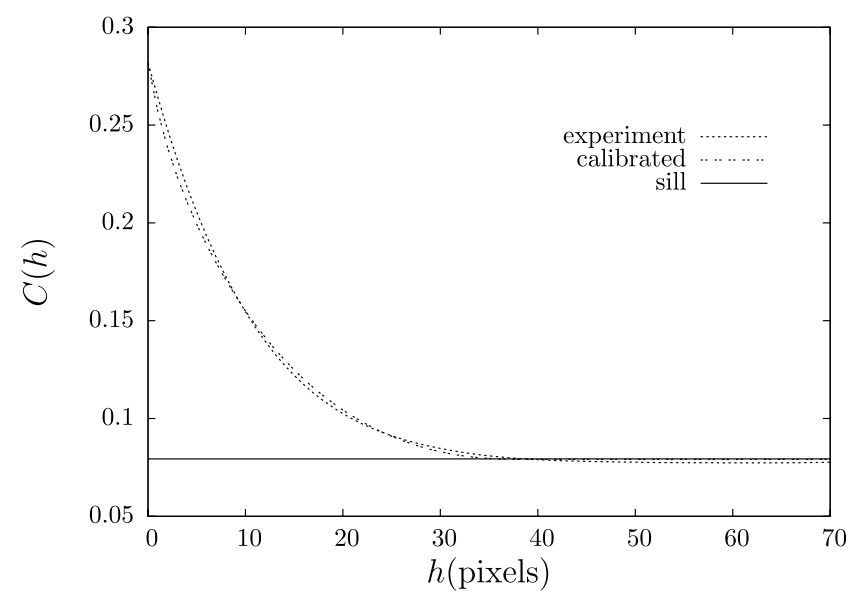

Fig. 26. The analytic covariance calibrated on the experimental data of the material $M_{2}$.

\section{Analytic fitting from the covariance (step 1)}

Figure 26 shows the analytic covariance fitted on the experimental data for material $M_{2}$. Table 1 gives the two sets of parameters identified for both materials, $M_{1}$ and $M_{2}$. Special attention is focused on the value obtained for the apparent volume fraction of the particles in the thickness $\left(V v_{p}^{\mathrm{app}}\right)$ and the corrected volume fraction for a zero thickness slice $\left(V v_{p}\right)$. The resulting volume fraction of carbon black is 0.1434 and 0.1442 for the materials $M_{1}$ and $M_{2}$, respectively. The radius of spherical particles is approximatively the same for both materials. In the following, only the radius of spherical inclusions and the volume fractions of the three scales are retained for the final identification of the set of parameters on the simulated TEM observations (step 2).

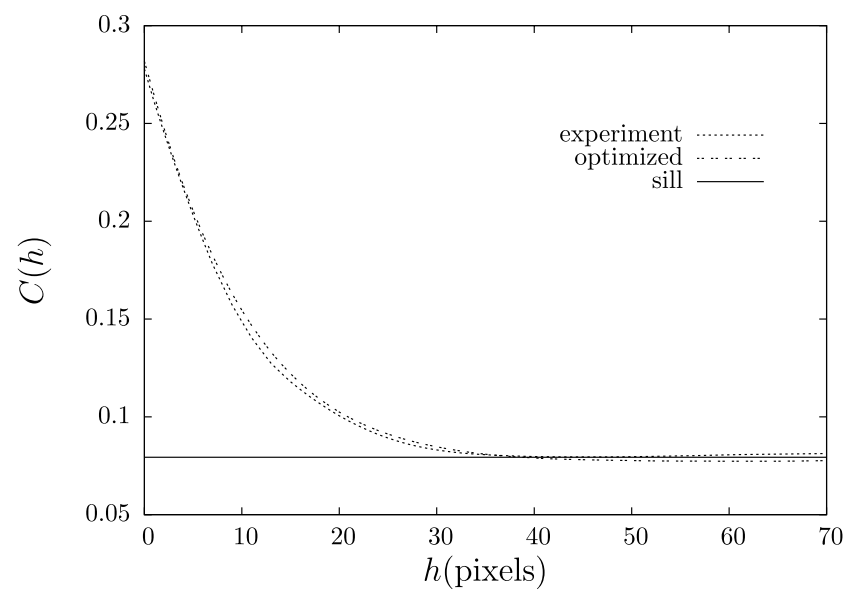

Fig. 27. The covariance on simulated microstructures optimized on the experimental data of the material $M_{2}$.

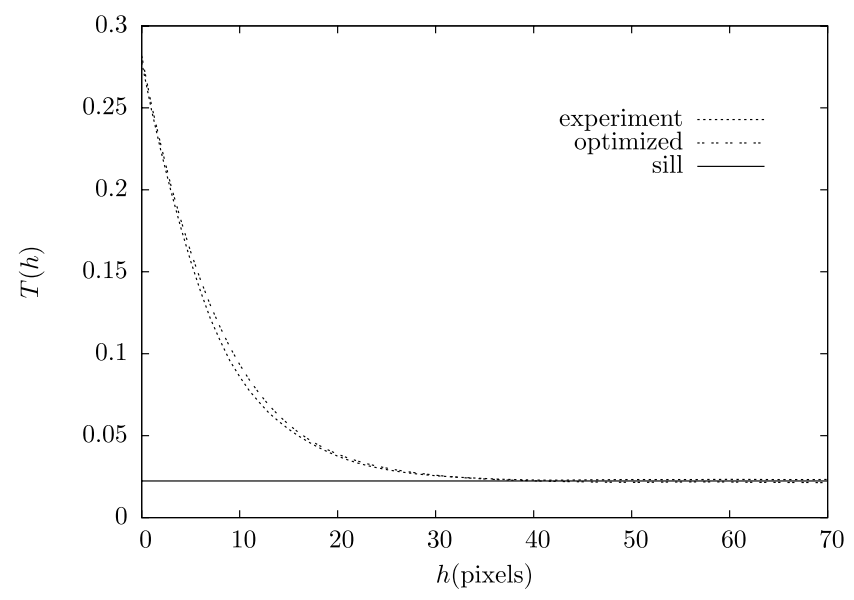

Fig. 28. The third-order moment on simulated microstructures optimized on the experimental data of the material $M_{2}$.

\section{Identification on simulations of microstructure (step 2)}

Figures 27-29 illustrate the identified covariance, third-order moment and closing curve respectively for the material $M_{2}$. Figure 30 shows the evolution of the error as a function of the number of iterations of the optimization computation. The error is the sum of the three least square norms, for both statistical moments and closing curve. For each criterion, the least square norm is expressed as follows:

$$
\frac{1}{2}(f(x)-y(x))^{\mathrm{T}} W(f(x)-y(x))
$$

where $f(x)$ is the $(N \times 1)$ matrix of the simulated data. $y(x)$ is the $(N \times 1)$ matrix of the experimental data. Finally, $W$ is the positive diagonal $(N \times N)$ weight matrix, $N$ being the number of points which are compared in the calculation of the least square norm. Here the number of points is the same 


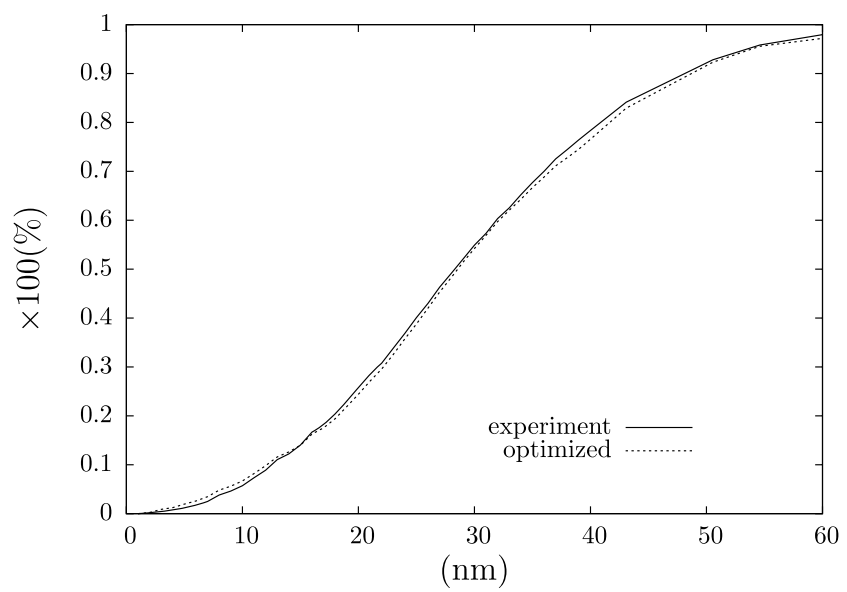

Fig. 29. The closing curve on simulated microstructures optimized on the experimental data of the material $M_{2}$.

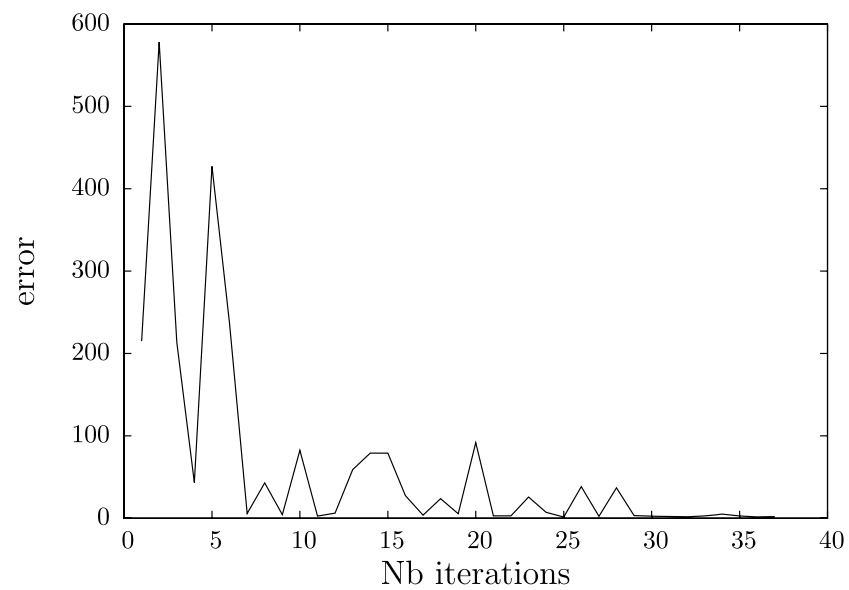

Fig. 30. The error as a function of the number of iteration in the optimization computation for the material $M_{2}$.

for the three criteria, and the weight is equal to 30000 in the case of the statistical moments and 20000 for the closing curve. Indeed, the statistical moments gives a more accurate information on the morphology than the closing curve.

The Table 2 gives the final set of four parameters optimized for both materials. Figures 31 and 32 show a simulated TEM image of materials $M_{2}$ and $M_{1}$, respectively. For each material, the aggregates and the matrix zones between aggregates are definitely well represented, as compared to binary TEM images. Regarding the mixing time, the distribution of carbon black fillers of material $M_{1}$ is less homogeneous than the one of material $M_{2}$. Hence, the simulations of microstructure of material $M_{1}$ show continuous paths of the carbon black fillers from one side of the simulation to another contrary to the material $M_{2}$. This continuous path is called a percolated path of the fillers in the simulation. Figure 33(a) illustrates a percolated three-dimensional simulation of the

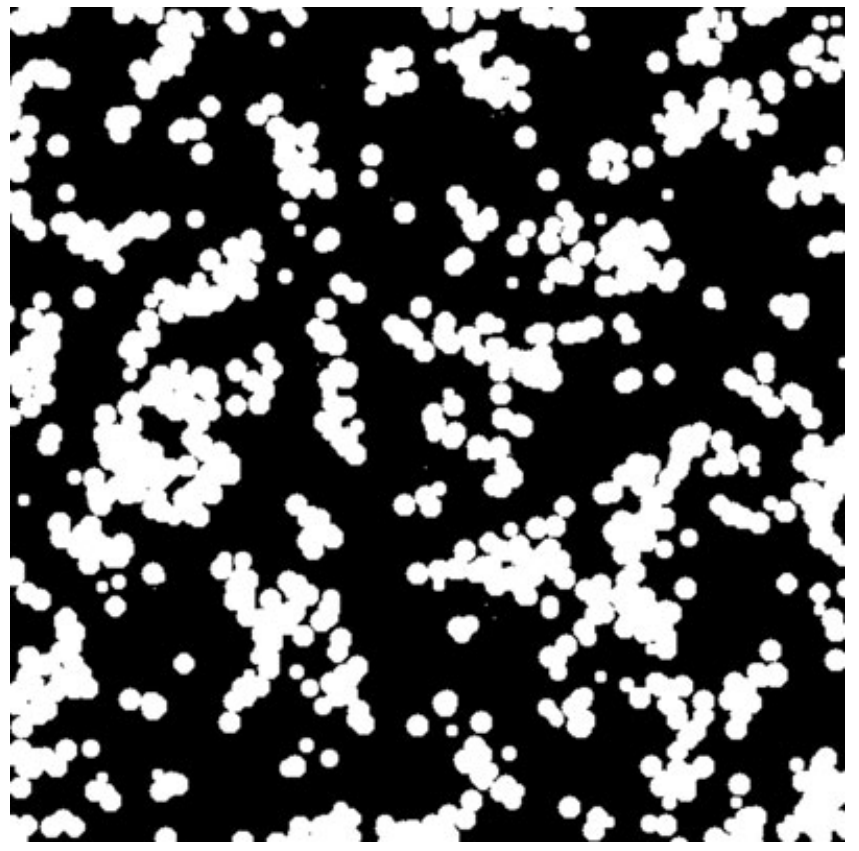

Fig. 31. A numerical TEM image of the material $M_{2}$.

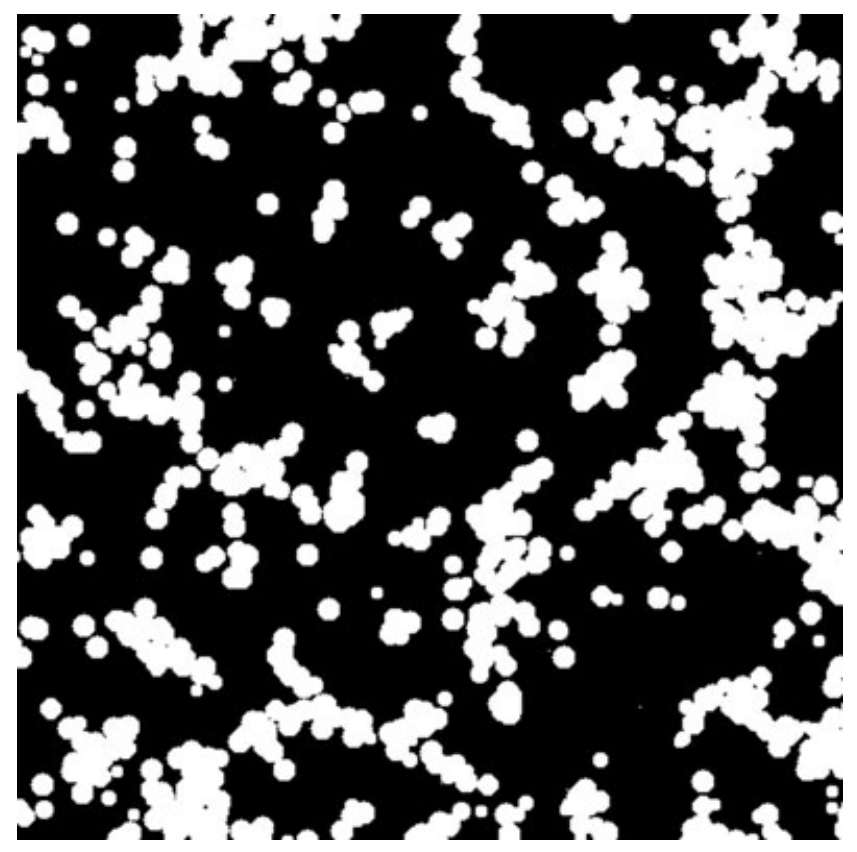

Fig. 32. A numerical TEM image of the material $M_{1}$.

microstructure of the material $M_{2}$, where each aggregate is labelled by a colour. Figure 33(b) shows the large percolating network in the simulation. The percolation of the fillers can be a useful criterion to validate the identification of the model for a material. The next section deals with the validation of this identification for both materials regarding the global percolation criterion. 
(a)

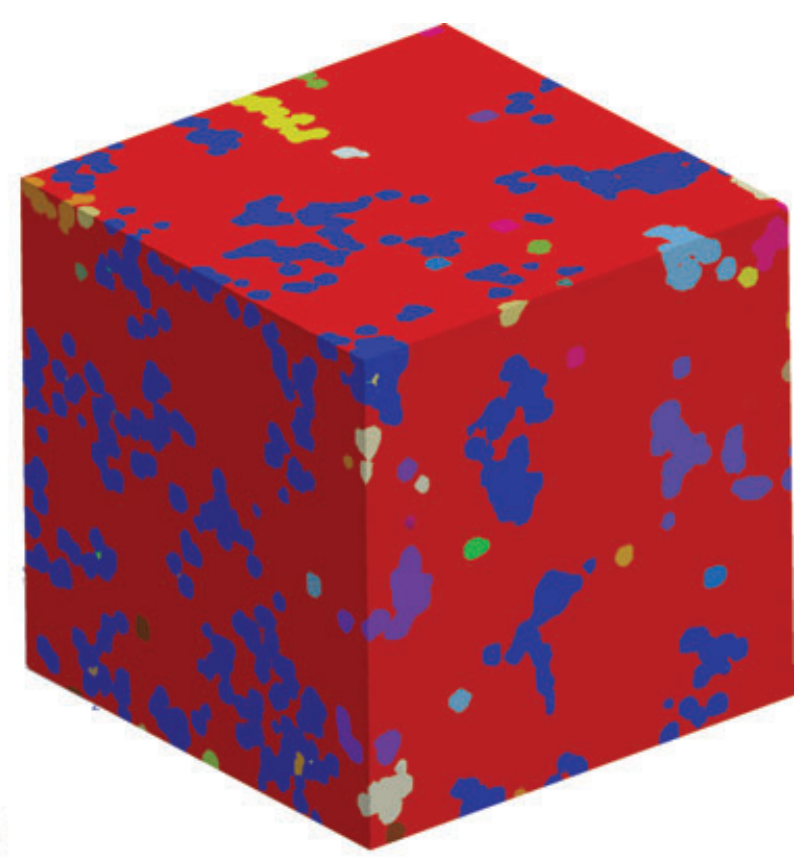

(b)

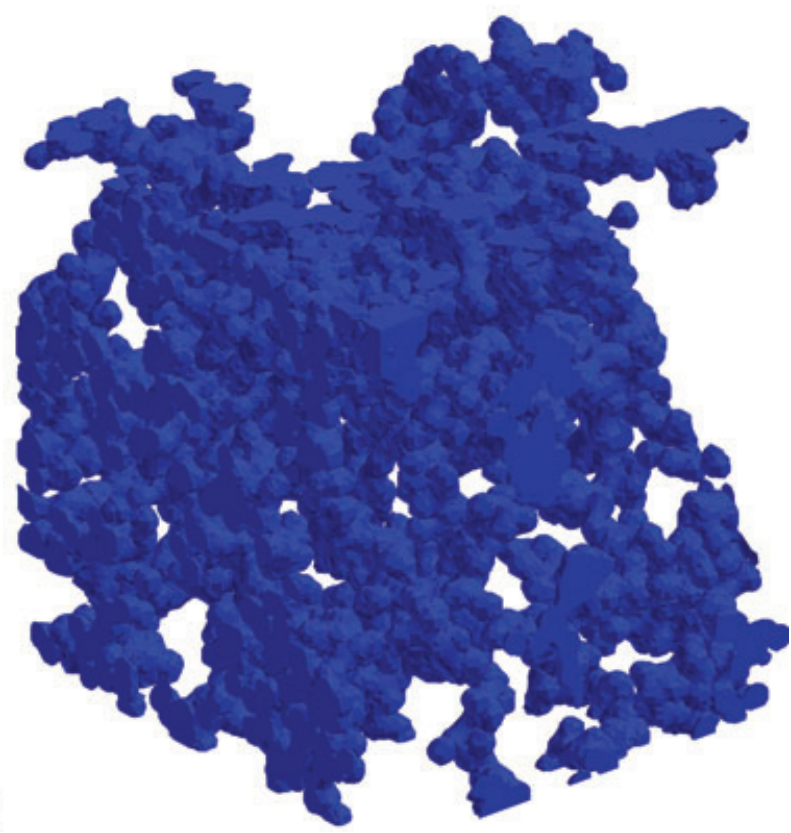

Fig. 33. A three-dimensional simulation of the material $M_{1}$ with the different aggregates (a) and the percolating network (b).

\section{Discussion: validation of the model according to a percolation criterion}

\section{Percolation behaviours}

For heterogeneous media the observation of the percolation behaviour of fillers in the matrix enables one to better understand the relation between the material physical behaviour and the microstructure. In Kanit et al. (2006), two families of percolation behaviour are introduced, the geometrical percolation and the mechanical one. For a two phase material, a phase is considered percolating geometrically when a continuous path of this phase can be drawn throughout a specimen. A heterogeneous loaded material is mechanically percolating when a continuous path of the same mechanical behaviour can be found throughout a specimen. In practice, regions with the same material behaviour are defined according to a threshold on a mechanical property. As a matter of fact, every geometrically percolating specimen is also mechanically percolating. In the case of a geometrically nonpercolating specimen, the elements belonging to the phase can be close enough so that the mechanical local behaviour of the second phase in between has the same behaviour as the first phase. In this work, the geometrically percolation behaviour is studied for the simulated microstructures. The mechanically percolation behaviour is discussed in (Jean et al., accepted) along with the well- known stress amplification of this rubber composite.

\section{Geometrical percolation}

Several experimental and numerical techniques can be used to measure geometrical percolation behaviour in materials. Two kinds of geometrical percolation behaviour can be defined: the global percolation and the local one. The global percolation concept is widely used. A simulation is said to be percolating when a continuous path of its fillers connects one face to the opposite one. The local percolation can be defined in globally percolating or non-percolating simulation. The local percolation concept is related to the length of percolating paths in the material for small scales. In practice, each path length of one phase through the complementary one is measured. This local measurement gives relevant pieces of information on the local phase arrangement and therefore the local mechanical behaviour.

For the rubber with carbon black fillers studied in this paper (Pécastaings, 2005) measured the percolating path in thick slices around $200 \mathrm{~nm}$ using a resiscope. This experimental technique consists in measuring the difference of electric potential between the two sides of the slice crossed by an electrical current. These measurements depend strongly on the experimental conditions. For instance a local difference of thickness of the slice due to the cutting process has a strong influence on the difference of electric potential. Nevertheless, they enable us to give trends when comparing materials. The local percolation was studied by estimation of percolation paths in simulated slices of both materials with increasing thickness. Up to $200 \mathrm{~nm}$ in thickness, the observation from resiscope and from simulations on both materials are in agreement with the global percolation. However, the decrease of the amount of the connected area with the thickness is stronger on the experimental observations made with the resiscope (Jean, 2009). Due to the lack of robustness of the 


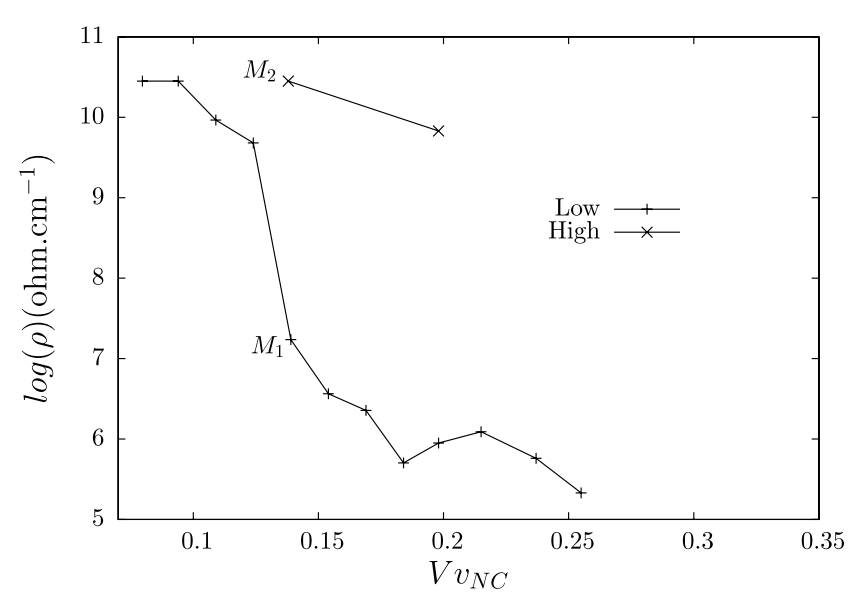

Fig. 34. Experiment resistivity of two set of materials.

local measurement, the global percolation measurement is chosen to validate the model.

\section{Validation}

Figure 34 illustrates the evolution of the resistivity (in logarithm scale) as a function of the volume fraction of fillers in material for two mixing times. The materials $M_{1}$ and $M_{2}$ are pointed on the graph for a volume fraction that is equal to $14 \%$. For the low mixing time $\left(M_{1}\right)$, the resistivity decreases significantly with the volume fraction of fillers increases. This phenomenon is typically encountered for a percolating threshold. Here, the material $M_{1}$ is considered to percolate regarding its resistivity. Contrary to the material $M_{1}$, the material $M_{2}$ does not percolate because its resistivity is high. These experimental results seem are in agreement with the simulations obtained in the Section 'Results'.

To validate the model capability to account for the global percolation, a quantitative study is carried out. This study consists in determining the proportion of percolating realizations for a given size of simulation. Here, a simulated material is considered percolating when more than $50 \%$ of simulations for a given size percolate. During the simulation of a realization of microstructure, each aggregate is labelled. A realization is considered to percolate when a same label can be found on two opposite sides of the realization. Figure 35 shows the evolution of percolating proportions of simulations as a function of the simulation length for both materials, $M_{1}$ and $M_{2}$. For a given size of simulation, 10 realizations were generated. The material $M_{2}$ percolates for sizes of simulation smaller than $700 \mathrm{~nm}$ which corresponds to the size of the largest aggregates in the material. For larger sizes of simulations, the material $M_{2}$ does not percolate. On the opposite, the material $M_{1}$ always percolates for every simulation sizes. These results are in agreement with the experimental ones. Thus, the model of microstructure associated with the method of identification proposed in this

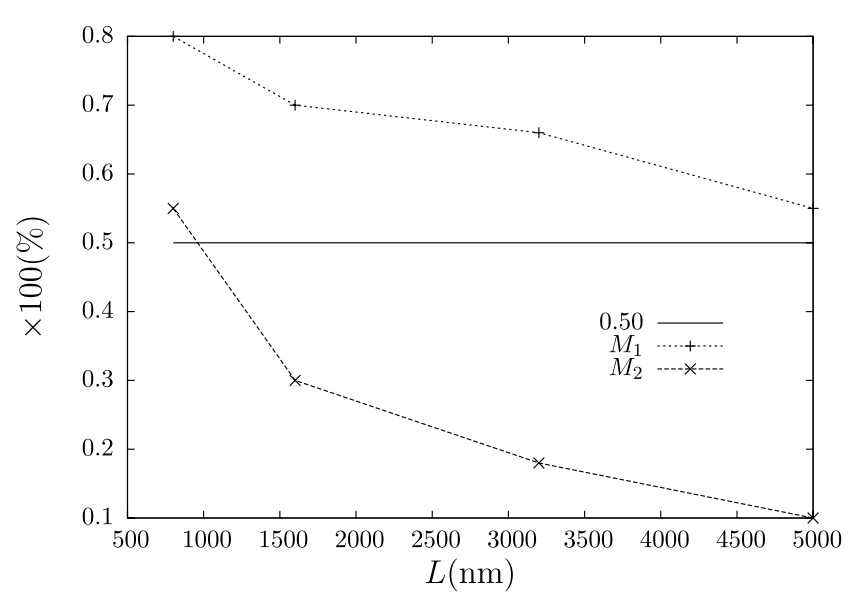

Fig. 35. Proportions of percolating simulations of microstructures as function of the size of the simulation ( $L$ in nanometres) for the materials $M_{1}$ and $M_{2}$.

paper enables us to account for the morphology of the fillers at large scales and is validated from a macroscopic percolation criterion.

\section{Conclusion}

An original method was proposed in this paper to model the multiscale distribution of carbon black fillers in an elastomeric matrix. A mathematical model was presented to take into account all the scales in the microstructure, the particle, the aggregate and the matrix. This model was identified from the statistical moments and morphological data simulating numerical TEM images. This method is generic enough to be used for others materials with any geometry.

Two materials with the same volume fraction of fillers but with a different mixing time were identified according to the method proposed in the paper. Due to the difference of mixing time, the TEM observations have shown clearly that the dispersion of fillers of one material is less homogeneous. One of the main question addressed in this paper concerns the capability of the proposed multiscale model associated with the original identification method to predict the strong difference of the three-dimensional morphology between both material. The global percolation criteria has been retained to validate the model.

Regarding the measurements of the resistivity of both materials, only one material percolates. The measurements of the proportion of percolating realizations for increasing sizes of simulations was in agreement with the experimental results. It has thus proved the relevance and the robustness of the strategy presented in this paper to summarize the microstructure by a multiscale model of random set.

A further validation was made by comparison of the elastic shear modulus of materials $M_{1}$ and $M_{2}$ from their microstructure to the macroscopic measurements (Jean, 2009; Jean et al., 2010). Finally, it will be possible to use the 
morphological model to propose new materials with improved properties.

\section{Acknowledgements}

The authors thank the Research Technology Center of Michelin for their financial and Scientific support.

\section{References}

Barbe, F., Decker, L., Jeulin, D. \& Cailletaud, G. (2001) Intergranular and intragranular behavior of polycrystalline aggregates. Part 1: FE model. Int. J. Plasticity 17, 513-536.

Bergstrom, J. \& Boyce, M.(1998) Constitutive modelling of the large strain time-dependent behavior of elastomers.J. Mech. Phys. Solids 46, 931954.

CMM/ARMINES/MinesParisTech/TRANSVALOR (2002) Micromorph, image analysis \& mathematical morphology. http://cmm.ensmp.fr/ Micromorph/.

Delarue, A. (2001) Prévision du comportement électromagnétique de matériaux composites partir de leur mode d'élaboration et de leur morphologie. PhD thesis, École des Mines de Paris.

Donnet, J. (2003) Nano and microcomposites of polymers elastomers and their reinforcement. Compos. Sci. Technol. 63, 1085-1088.

Gérard, C., N'Guyen, F., Osipov, N., Cailletaud, G., Bornert, M. \& Caldemaison, D. (2009) Comparison of experimental results and finite element simulation of strain localization scheme under cyclic loading. Comput. Mater. Sci. 46, 755-760.

Hain, M. \& Wriggers, P. (2008) Computational homogenization of microstructural damage due to frost in hardened cement paste. Finite Elem. Anal. Des. 44, 233-244.

Jean, A. (2009) Etude d'un élastomère chargé, de sa nanostructure à son macro-comportement. PhD thesis, Ecole des Mines de Paris - PariTech.

Jean, A., Willot, F., Cantournet, S., Forest, S. \& Jeulin, D. (2010) Large scale computations of effective elastic properties of rubber with carbon black fillers. International Journal of Multiscale Computational Engineering. Accepted for Publication.

Jean, A., Jeulin, D., Cantournet, S., Forest, S. \& N'Guyen, F. (2007) Rubber with carbon black fillers: parameters identification of a multiscale nanostructure model. In Boukamel, A., Laiarinandrasana, L., S., M. \& Verron, E., editors, Constitutive Models for Rubber V. Proceedings of 5th European Conference on Consitutive Models for Rubber, ECCMR 2007, Paris, France, 4-7 September 2007, pp. 141-146, Taylor and Francis, London.

Jeulin, D. (1991) Modèles morphologiques de structures aléatoires et de changement d'échelle. Thèse de Doctorat d'Etat, Université de Caen, France.

Jeulin, D. \& Le Coënt, A. (1996) Morphological modeling of random composites. In Markov, K. Z., editor, Proceedings of the CMDS8 58 Conference, Varna, Bulgaria, 11-16 June, 1995, pp. 199-206. World Scientific Publishing Company.

Jha, V., Thomas, A., Fukahori, Y. \& Busfield, J. (2007) Micro-structural finite element modelling of the stifness of filled elastomers: the effect of filler number, shape and position in the rubber matrix. In Boukamel, A.,
Laiarinandrasana, L., S., M. \& Verron, E., editors, Constitutive Models for Rubber V. Proceedings of 5 th European Conference on Consitutive Models for Rubber, ECCMR 2007, Paris, France, 4-7 September 2007, pp. 141-146, Taylor and Francis, London.

Kanit, T., N’Guyen, F., Forest, S., Jeulin, D., Reed, M. \& Singleton, S. (2006) Apparent and effective physical properties of heterogeneous materials: representative of samples of two materials from food industry. Comput. Methods Appl. Mech. Eng. 195, 3960-3982.

Laiarinandrasana, L., Jean, A., Jeulin, D.S. \& Forest, S. (2009) Modelling the effects of various contents of fillers on the relaxation rate of filled rubbers. In Constitutive Models for Rubber VI. Proceedings of 6th European Conference on Consitutive Models for Rubber, ECCMR 2009, Dresden, Germany, 7-10 September 2009, Taylor and Francis, London.

Lorensen, W. \& Cline, H., editors (1987) Marching cubes: a high resolution 3D surface reconstruction algorithm. Computer Graphics (Proceedings of SIGGRAPH '87, 21(4), 163-169.

Madi, K., Forest, S., Boussuge, M., Gailliègue, S., Lataste, E., Buffière, J.-Y., Bernard, D. \& Jeulin, D. (2007) Finite element simulations of the deformation of fused-cast refractories based on x-ray computed tomography. Comput. Mater. Sci. 39, 224-229.

Matheron, G. (1967) Éléments pour une théorie des milieux poreux. Paris, Masson.

Mines ParisTech, ONERA, N. (2003) Zset. http://zebulon.onera.fr/ English/index.html.

Moreaud, M. \& Jeulin, D. (2005) Multi-Scale Simulation of Random Spheres Aggregates: Application to Nanocomposites, pp. 341-348, Zakopane, Poland. ECS 9.

Naito, M., Muraoka, K., Azuma, K. \& Tomita, Y. (2007) 3D modeling and simulation of micro to macroscopic deformation behavior of filled rubber. In Boukamel, A., Laiarinandrasana, L., S., M. \& Verron, E., editors, Constitutive Models for Rubber V. Proceedings of 5th European Conference on Consitutive Models for Rubber, ECCMR 2007, Paris, France, 4-7 September 2007, pp. 141-146, Taylor and Francis, London.

Osipov, N., Gourgues-Lorenzon, A.-F., Marini, B., Mounoury, V., N'Guyen, F., and Cailletaud, G. (2008) FE modelling of bainitic steels using crystal plasticity. Philos. Mag. 88, 3757-3777.

Payne, A. (1962) The dynamic properties of carbon black loaded natural rubber vulcanizates. II. J. Appl. Polym. Sci. VI(21), 368-372.

Pécastaings, G. (2005) Contribution l'étude et la modélisation de la mésostructure de composites polymères-noir de carbone. $\mathrm{PhD}$ thesis, Université Bordeaux I.

Sab, K. \& Boumediene, N. (2005) Periodization of random media and representative volume element size for linear composites. C. R. Acad. Sci. Paris 333, 187-195.

Savary, L., Jeulin, D. \& Thorel, A. (1999) Morphological analysis of carbonpolymer composite materials from thick section. Acta Stereol. 18, 297303.

Serra, J. (1982) Image Analysis and Mathematical Morphology. Academic Press, London.

Weaire, D. (2008) Kelvin's foam structure: a commentary. Philos. Mag. Lett. 88, 91-102.

Zeman, J. \& Sejnoha, M. (2001) Numerical evaluation of effective elastic properties of graphit fibre tow impregnated by polymer matrix. J. Mech. Phys. Solids 49, 69-90. 